ARTICLE

https://doi.org/10.1038/s41467-019-09598-9

\title{
The class 3 PI3K coordinates autophagy and mitochondrial lipid catabolism by controlling nuclear receptor PPAR $\alpha$
}

\author{
Anton lershov (10) 1,2,3, Ivan Nemazanyy 1,2,3,4, Chantal Alkhoury ${ }^{1,2,3}$, Muriel Girard ${ }^{1,2,3,5}$, Esther Barth ${ }^{6}$, \\ Nicolas Cagnard ${ }^{7}$, Alexandra Montagner ${ }^{8}$, Dominique Chretien ${ }^{9,10}$, Elena I. Rugarli ${ }^{6}$, Herve Guillou ${ }^{11}$, \\ Mario Pende ${ }^{1,2,3}$ \& Ganna Panasyuk (i) 1,2,3
}

The class 3 phosphoinositide 3-kinase (PI3K) is required for lysosomal degradation by autophagy and vesicular trafficking, assuring nutrient availability. Mitochondrial lipid catabolism is another energy source. Autophagy and mitochondrial metabolism are transcriptionally controlled by nutrient sensing nuclear receptors. However, the class 3 PI3K contribution to this regulation is unknown. We show that liver-specific inactivation of Vps15, the essential regulatory subunit of the class 3 PI3K, elicits mitochondrial depletion and failure to oxidize fatty acids. Mechanistically, transcriptional activity of Peroxisome Proliferator Activated Receptor alpha (PPAR $\alpha$ ), a nuclear receptor orchestrating lipid catabolism, is blunted in Vps15-deficient livers. We find PPAR $\alpha$ repressors Histone Deacetylase 3 (Hdac3) and Nuclear receptor co-repressor 1 (NCoR1) accumulated in Vps15-deficient livers due to defective autophagy. Activation of PPAR $\alpha$ or inhibition of Hdac3 restored mitochondrial biogenesis and lipid oxidation in Vps15-deficient hepatocytes. These findings reveal roles for the class 3 PI3K and autophagy in transcriptional coordination of mitochondrial metabolism.

\footnotetext{
${ }^{1}$ Institut Necker-Enfants Malades (INEM), 75014 Paris, France. ${ }^{2}$ INSERM U1151/CNRS UMR 8253, 75014 Paris, France. ${ }^{3}$ Université Paris Descartes, Sorbonne Paris Cité, 75006 Paris, France. ${ }^{4}$ Platform for Metabolic Analyses, Structure Fédérative de Recherche Necker, INSERM US24/CNRS UMS 3633, 75014 Paris, France. ${ }^{5}$ Pediatric Hepatology Unit, Hôpital Necker-Enfants Malades, Assistance Publique-Hôpitaux de Paris, Paris 75015, France. ${ }^{6}$ Institute for Genetics, Cologne Excellence Cluster on Cellular Stress Responses in Aging-Associated Diseases (CECAD), University of Cologne, 50674 Cologne, Germany. ${ }^{7}$ Plateforme Bio-informatique, Université Paris Descartes, Structure Fédérative de Recherche Necker, INSERM US24/CNRS UMS 3633, Paris 75015, France. ${ }^{8}$ INSERM U1048, Université Paul Sabatier, Toulouse 31432, France. ${ }^{9}$ INSERM UMR1141, Hôpital Robert Debré, Paris 75019 , France. ${ }^{10}$ Université Paris 7, Faculté de Médecine Denis Diderot, Paris 75019, France. ${ }^{11}$ Toxalim, Université de Toulouse, INRA, ENVT, INP-Purpan, UPS, Toulouse 31027, France. These authors contributed equally: Anton lershov, Ivan Nemazanyy. Correspondence and requests for materials should be addressed to G.P. (email: ganna.panasyuk@inserm.fr)
} 
M etabolic homeostasis is achieved by coordinated synthesis and degradation of macromolecules. To this end, lysosomal degradation by autophagy and mitochondrial catabolism are essential in periods of nutrient shortage such as fasting ${ }^{1,2}$. Studies in mouse mutants have demonstrated the requirement of autophagy in the adaptation to fasting ${ }^{3-5}$. Notably, mitochondria contribute to energy-demanding autophagosome formation and autophagic flux by providing ATP and lipids, such as phosphatidylethanolamine ${ }^{6-8}$. On the other hand, autophagy supplies substrates for the oxidation reactions that take place in mitochondria, such as fatty acids and amino acids through lipophagy and proteolysis, respectively ${ }^{2,9,10}$. Autophagic clearance of defective mitochondria also assures mitochondrial quality control ${ }^{11}$. Consistently, the accumulation of dysfunctional swollen mitochondria is commonly found in autophagy deficient cells $s^{3,12-15}$. In fasting, both autophagy and mitochondrial activity are transcriptionally coordinated. This coordination, in liver tissue, relies on the activation of basic helix-loop-helix transcription factor EB (TFEB) and nutrient sensor transcription factors of the nuclear receptor superfamily including PPARa ${ }^{16-20}$. Of note, TFEB and the PPARa/PGC1a transcriptional complex play overlapping roles in transcriptional control of the autophagy related gene network (Coordinated Lysosomal Expression and Regulation (CLEAR) network) ${ }^{18-21}$. In addition, PPARa activates fatty acid $\beta$-oxidation (FAO) and ketogenesis, while PGC1a is known to be a master regulator of mitochondrial biogenesis. PGC1a co-activates nuclear respiratory factors (NRFs) and estrogen-related receptor (ERRs) transcription factors to promote the expression of proteins for mitochondrial DNA replication, mitochondrial RNA transcription as well as factors for mitochondrial maintenance ${ }^{22}$. Although previous studies in autophagy-deficient hepatic mutants of Atg5 and Fip200 have suggested that PPARa transcriptional responses in lipid catabolism might be suppressed, a mechanistic understanding of this dysfunction is lacking ${ }^{10,23,24}$.

Within the autophagy network, the class 3 PI3K, present in all eukaryotes, plays a central role ${ }^{25}$. It functions as an obligate complex of a regulatory Vps15 subunit and a catalytic lipid kinase Vps34 subunit. Vps15 is a putative serine/threonine protein kinase required for Vps34 stability and activity ${ }^{26}$. The lipid kinase activity of $\mathrm{Vps} 34$ is a major source of the secondary messenger phosphatidylinositol 3-phosphate (PI3P) ${ }^{27}$. PI3P serves as a docking signal for proteins containing PI3P binding domains, such as FYVE or $\mathrm{PX}^{28}$. In the cell, PI3P is generated at phagophore membrane during autophagy initiation. It is also essential for endosomal sorting of plasma membrane proteins internalised by endocytosis, and it is required for delivery of hydrolases into the lysosome. In all these distinct processes, PI3P nucleates protein scaffolds to promote autophagic flux and vesicular trafficking towards the lysosome, thus placing the class 3 PI3K in control of fundamental nutrient acquisition pathways. To activate these distinct processes, the class 3 PI3K engages in different protein complexes ${ }^{25}$. To this end, the binding of Atg14-related protein (Atg14) or ultraviolet radiation resistance-associated gene protein (UVRAG) to the Vps34/Vps15 complex is mutually exclusive ${ }^{29,30}$. Atg14 stimulates Vps34 activity at phagophore membranes and is required for autophagy initiation in response to nutrient withdrawal, while the UVRAG-containing complex is implicated in endosome and autophagosome maturation ${ }^{25,29,30}$.

Gene knockouts of either Vps34 or Vps15 revealed that the class 3 PI3K activity is indispensable for embryogenesis and organ function $^{31,32}$. The implication of the class 3 PI3K in metabolic homeostasis is backed by the phenotypes of tissue-specific mutants. As we have previously reported, deletion of Vps15 in the liver results in defective glucose homeostasis due to increased insulin receptor signalling ${ }^{33}$. Recent work has further demonstrated that even a partial inactivation of the class 3 PI3K in a mouse model of a heterozygous Vps34 lipid kinase knock-in expression, enhances insulin sensitivity and glucose tolerance ${ }^{34}$. However, unlike its role in autophagy, the mechanistic implication of the class $3 \mathrm{PI} 3 \mathrm{~K}$ signalling in mitochondrial function is not fully understood. Similar to mutants of essential autophagy genes, decreased mitochondrial respiration was found in Vps34null cells ${ }^{34}$. Yet, unlike in Atg-mutants, small-sized mitochondria were traced in Vps34-null hepatocytes suggesting a possible effect on mitochondrial biogenesis ${ }^{13}$. These observations suggest that the class $3 \mathrm{PI} 3 \mathrm{~K}$ might contribute to the transcriptional control of mitochondrial metabolism, a hypothesis that has not yet been addressed experimentally. Here we show that hepatic expression of the class $3 \mathrm{PI} 3 \mathrm{~K}$ is essential for metabolic adaptation to starvation in the liver through the control of PPAR $\alpha$ transcriptional activity. We demonstrate that the availability of PPAR $\alpha$ ligands and expression of co-activator PGC1a, as well as levels of PPARa repressors $\mathrm{NCoR} 1$ and $\mathrm{Hdac} 3$ are affected in mice with liverspecific inactivation of Vps15. Mechanistically, we show that Hdac3 and NCoR1 repressors interact with Autophagy-related protein 8 (Atg8)-like proteins and degrade in lysosome, a mechanism that is non-functional in autophagy deficient Vps15-null hepatocytes. Notably, the pharmacologic inhibition of histone deacetylases and administration of a PPARa synthetic ligand was sufficient to significantly improve mitochondrial function in Vps15-null livers. Finally, we show that, in addition to its known role in transcriptional control of ketogenesis and FAO, PPAR $\alpha$ acts upstream of mitochondrial biogenesis in the liver. Ultimately, we propose that the class 3 PI3K acts upstream of nuclear receptors and exerts a broad transcriptional control in the liver to match autophagic activity with mitochondrial metabolism during fasting.

\section{Results}

Mitochondria are dysfunctional in Vps15-LKO mice. In our recent work, we demonstrated that deletion of the regulatory subunit of the class $3 \mathrm{PI} 3 \mathrm{~K}, \mathrm{Vps} 15$, in hepatocytes provoked metabolic rearrangements that were mirrored by modified transcription of metabolic enzymes ${ }^{33}$. It led us to hypothesise that the class 3 PI3K exerts a broad transcriptional control in liver. To test this, we performed microarray analyses upon inactivation of the class 3 PI3K. To avoid a possible adaptation to a chronic loss of the class $3 \mathrm{PI} 3 \mathrm{~K}, \mathrm{Vps} 15$ was acutely depleted in livers of $\mathrm{Vps} 15^{\mathrm{f} / \mathrm{f}}$ mice using adenoviral vectors expressing Cre recombinase or GFP protein as a control. This acute liver-specific deletion of Vps15 resulted in significant liver hypertrophy and autophagy block $^{33}$. Following analyses demonstrated that transcript levels of 2693 unique genes were modified more than 1.5-fold. Among those $1225(45 \%)$ were significantly downregulated and 1471 (55\%) were significantly upregulated (Supplementary Data 1). The subsequent Gene Ontology (GO) pathway analyses among the downregulated genes showed an enrichment of mitochondria, peroxisome and metabolic processes associated with those organelles; notably lipid metabolism (Fig. 1a; Supplementary Data 2). The GO analyses among the upregulated genes demonstrated that, consistent with the defective autophagy, there was an enrichment of oxidative stress response (Supplementary Fig. 1a, Supplementary Data 2). Further analyses by real-time quantitative PCR (RT-qPCR) of liver-specific Vps15-deficient AlbCre ${ }^{+}$; $\mathrm{Vps} 15^{\mathrm{f} / \mathrm{f}}$ mice $^{33}$, hereafter referred to as Vps15-LKO, confirmed these initial observations. Notably, marked oxidative stress in liver tissue of Vps15-LKO mice was evidenced by increased expression and activity of key transcription factor for antioxidant gene expression induction Nuclear factor erythroid 2-related factor 2 (Nrf2) (Supplementary Fig. 1b-d). Remarkably, among 
a

Number of downregulated genes \%



d



b

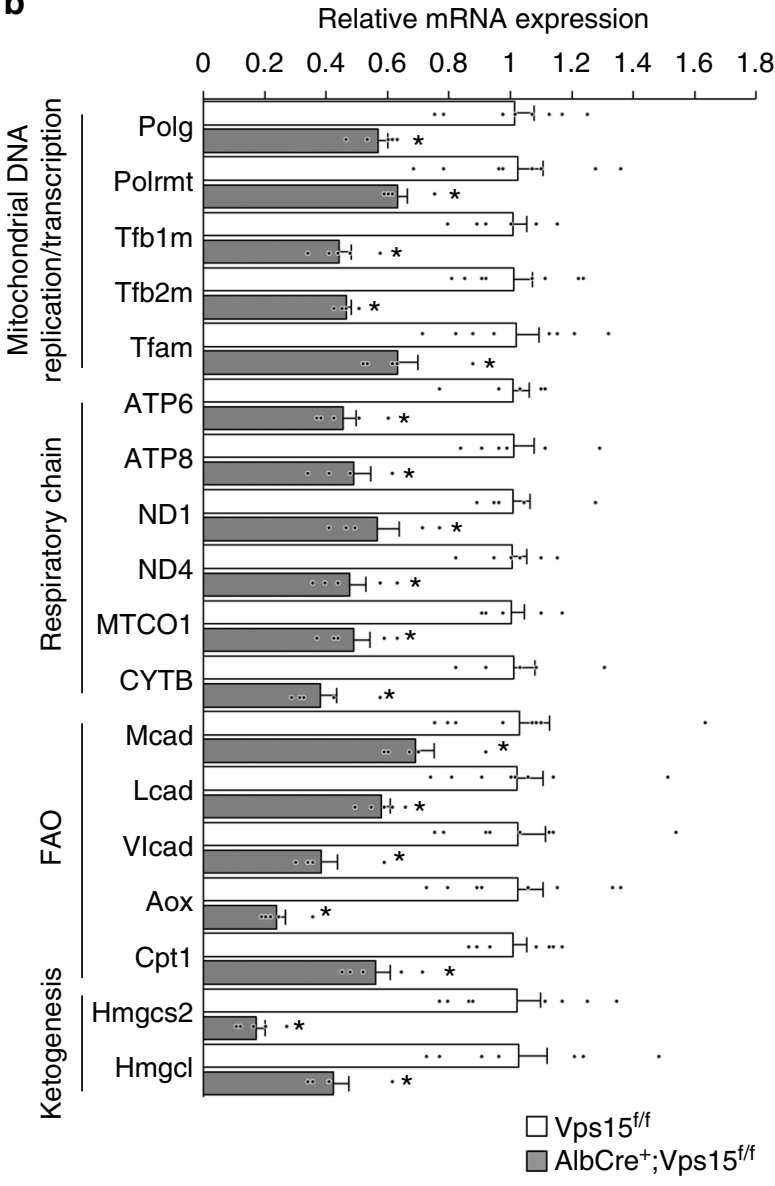

e
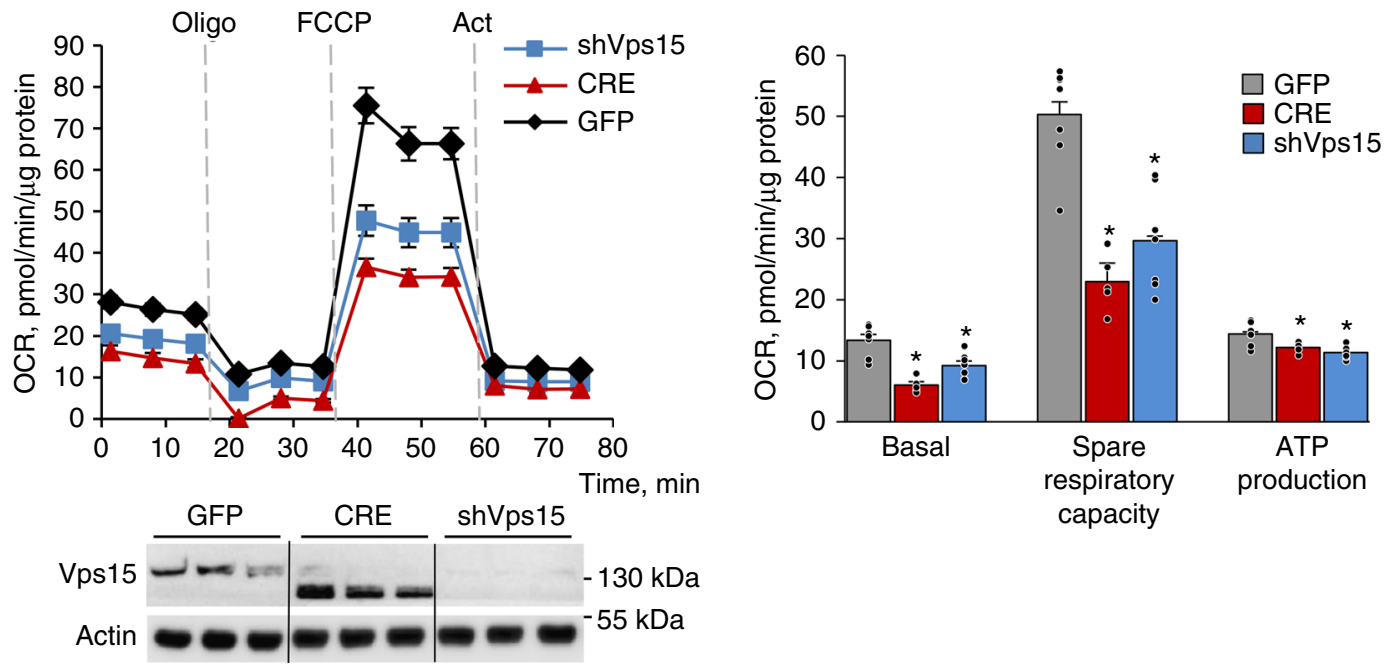

the downregulated genes, the transcript levels of enzymes involved in FAO; ketogenesis; and the expression of both nuclear and mitochondrial genome-encoded genes implicated in mitochondrial function and biogenesis were significantly decreased (by $40-80 \%$ ) in liver tissues of Vps15 mutants (Fig. 1b). These defects at the transcriptional level were accompanied by mitochondria depletion as seen by decreased Tom 40 and cytochrome c staining in primary hepatocytes isolated from Vps15-LKO mice and significantly decreased mtDNA content in liver tissue of Vps15-LKO mice (Fig. 1c, Supplementary Fig. 2a and 2b). Mitochondrial dysfunction was also evidenced by decreased total

respiratory chain complexes activity measured in liver extracts of Vps15-LKO mice (Fig. 1d). The later was due to a $20-60 \%$ decrease in activity of individual respiratory chain complexes (Supplementary Fig. 2c). This decreased enzymatic activity of respiratory chain complexes was accompanied by increased activity of lactate dehydrogenase in liver tissue of Vps15-LKO mice suggesting activated glycolysis (Supplementary Fig. 2d). These findings in liver tissue were also corroborated by analyses in primary hepatocytes. The metabolic profiling of mitochondria using Seahorse Bioanalyzer showed that acute depletion of Vps15 using specific shRNA or by expressing Cre recombinase in 
Fig. 1 Mitochondrial gene transcription programme is defective in Vps15-LKO mice. a Functional annotation clustering by Database for Annotation, Visualization and Integrated Discovery (DAVID) bioinformatic tool of significantly downregulated genes in the microarray analyses of liver tissue after acute Vps15 depletion. Percentage of genes among downregulated genes attributed to listed processes is indicated. Liver tissue was harvested ten days after transduction with adenoviral vectors expressing Cre recombinase to deplete Vps15 or GFP control protein. Significantly modified genes are listed in Supplementary Data 1. Functional annotation clustering results are listed in Supplementary Data 2. b Relative transcript levels of genes implicated in mitochondrial DNA replication, transcription, respiratory chain subunits and metabolic enzymes in livers of control and Vps15-LKO mice analysed by RT-qPCR. Data are means \pm SEM ( $n=6-8$ for $V p s 15^{f / f}, n=5$ for AlbCre ${ }^{+} ; V_{p s} 15^{f / f}, P<0.05^{*}$ : vs Vps15/f mice, two-tailed, unpaired Student's $t$ test). c Immunofluorescent analyses of Tom 40 in control and Vps15-null primary hepatocytes. Cells were PFA fixed and stained with anti-Tom 40 antibody, secondary anti-rabbit IgG Alexa Fluor 568 antibody was used for detection. Scale bar: $50 \mu \mathrm{m}$. d The total respiratory chain activity normalized to total protein content measured in liver tissue extracts of $\mathrm{Vps} 15^{\mathrm{f} / \mathrm{f}}$ and $\mathrm{AlbCre}{ }^{+} ; \mathrm{Vps} 15^{\mathrm{f} / \mathrm{f}}$ mice. Data are means $\pm \mathrm{SEM}\left(n=4\right.$ for $\mathrm{Vps} 15^{\mathrm{f} / \mathrm{f}}$ and $\mathrm{AlbCre}{ }^{+} ; \mathrm{Vps} 15^{\mathrm{f} / \mathrm{f}}$ $P<0.05^{*}$ : vs $V p s 15^{f / f}$, two-tailed, unpaired Student's $t$ test). e The oxygen consumption rate measured by SeaHorse Bioanalyzer in primary $V p s 15^{f / f}$ hepatocytes 48-h post-transduction with adenoviral vectors expressing GFP, Cre or shRNAVps15 under basal conditions (initial rates) and in response to sequential treatment with Oligomycin (respiration associated with ATP production), FCCP (maximal respiration), and Rotenone/Antimycin A (non-mitochondrial respiration). Dashed lines indicate the time of the addition of each reagent. Representative experiment of five independent hepatocyte cultures is presented. Quantification of basal respiration, ATP production and maximal respiratory capacity are shown on the graphs (right panel). Data are means \pm SEM, $P<0.05^{*}$ : vs GFP-infected primary hepatocytes, two-tailed, unpaired Student's $t$ test. Lower panel shows the control immunoblot analysis of total protein extracts of hepatocytes using indicated antibodies. The immunoblot with anti-actin antibody served as a loading control

$\mathrm{Vps} 15^{\mathrm{f} / \mathrm{f}}$ primary hepatocytes resulted in inhibition of basal and spare mitochondrial respiration as well as decreased ATP production capacity (Fig. 1e). Of note, the lower migrating band of $\mathrm{Vps} 15$ is a truncated non-functional form of the protein that is transcribed from the start codon in exon 4 upon effective Cre-mediated recombination in Vps15 gene locus ${ }^{31}$. Importantly, the inhibition of mitochondrial respiration and metabolic gene expression in Vps15-depleted hepatocytes was not due to expression of Cre-recombinase, as these effects were not observed in transduced primary hepatocytes isolated from wild-type mice (Supplementary Fig. 2e and 2f). Therefore, deletion of Vps15 in hepatocytes results in a transcriptional inhibition of mitochondrial maintenance program that manifests as mitochondrial dysfunction.

Fatty acid degradation is blocked in livers of Vps15-LKO mice. To get further insights in the metabolic rearrangements in Vps15LKO mice, the targeted metabolomics analyses by mass spectrometry were performed on liver tissue of $6-\mathrm{h}$ fasted animals. The pathway analyses of metabolomics data using MetaboAnalyst 4.0 tool suggested significant changes in lipid metabolism (Fig. 2a; Supplementary Data 3). Indeed, the long-chain-carnitine conjugates (C6-C16-carnitines) were accumulated in livers of Vps15LKO mice (Fig. 2b). This was paralleled by decreased levels of free fatty acids and lower levels of Acetyl-CoA in livers of Vps15-LKO mice (Fig. 2b). Importantly, in agreement with defective fatty acid degradation in mitochondria, measurements of the plasmatic levels of ketone body metabolite, hydroxybutyrate, revealed a 2.7fold lower levels in fasted Vps15-LKO mice (Fig. 2c). Altogether, metabolomics analyses advocate that Vps15 mutant hepatocytes do not sustain functional mitochondrial oxidation of fatty acids in fasting.

PPARa is inhibited in Vps15-null liver. The metabolic dysfunction of Vps15-LKO mice was reminiscent of a PPARa deficiency, whose transcriptional activity in the liver is essential for fatty acid uptake, transport, $\beta$-oxidation and ketogenesis ${ }^{35-37}$. Similar to reported mouse mutants of PPARa, Vps15-LKO mice were hypoketogenic (Fig. 2c) and hypoglycaemic ${ }^{33,35,36}$. Furthermore, bioinformatic analyses using EnrichR database of overrepresented transcription factors associated with differentially expressed genes in Vps15-null liver demonstrated proinflammatory and hematopoietic transcription factors on the top of the list for upregulated genes while RXR and PPARa as the most significantly associated with downregulated gene expression signature (Supplementary Fig. 3a). The likely involvement of PPARa in the phenotype of Vps15-LKO mice was also corroborated by hepatic gene expression profile in liver tissue upon acute deletion of Vps15, which unveiled lipid catabolism among the statistically enriched pathways (Fig. 1a). The comparative microarray analyses between Vps15-null livers and liver tissue from PPARa mutants (whole-body ${ }^{17}$ and hepatocyte-specific ${ }^{37}$ ) demonstrated a notable overlap in transcriptional responses (Fig. 2d, Supplementary Data 4). To address the status of PPARa in a condition of its maximal activation, Vps15-LKO mice were challenged by a 24-h fasting. The activation of PPARa in livers of control mice was evidenced by a induction of transcript levels of its bona fide targets in fatty acid transport to mitochondria, ketogenesis and fatty acid oxidation, Cpt2, Hmgcs2 and Aox, respectively (Fig. 2e). Importantly, their transcriptional induction in response to fasting was blunted in livers of Vps15-LKO mice (Fig. 2e). In agreement with its transcriptional inhibition, nuclear PPARa protein was depleted and its transcript levels downregulated in Vps15-null livers (Fig. 2f and Supplementary Fig. 3b). Proteosomal degradation is established mechanism of PPARa protein turnover ${ }^{38,39}$. In line with lower protein levels of PPARa protein, PPARa polyubiquitination was upregulated in livers of Vps15-null mice despite its lower protein expression detected in total extracts (Supplementary Fig. $3 \mathrm{c}$ and $3 \mathrm{~d}$ ). This was accompanied by increased in Vps15-LKO mice levels of Huwe1, a reported E3 ubiquitin ligase for PPARa, (Supplementary Fig. 3d) 39. Direct ligand binding controls PPARa protein expression, stability, and nuclear localization ${ }^{38,40}$. Fatty acids are known natural PPARa ligands ${ }^{41-44}$. They are delivered to the liver from adipose tissue upon fasting-induced lipolysis ${ }^{37}$, as well as being synthesized in hepatocytes de novo ${ }^{43}$. As expected, the 24-h fasting potently induced steatosis in the liver of control animals, showed as the pale colour of the organ and histological findings of vacuolation in hepatocytes (Supplementary Fig. $4 \mathrm{a}$ and $4 \mathrm{~b}$ ). Notably, these responses were not apparent in Vps15-LKO mice as their livers were already pale and hepatocytes were markedly vacuolated (Supplementary Fig. 4a and 4b). Biochemical analyses of triglyceride levels confirmed neutral lipid accumulation in liver tissue of control mice, a response that was blunted in Vps15-LKO mice (Supplementary Fig. 4c). The dampened response to fasting in livers of Vps15-LKO mice was also evidenced by unmodified liver hypertrophy, unlike decreased liver size in fasted control mice (Supplementary Fig. 4d). To get further insight in the lipid mobilisation in Vps15-LKO mice, the biochemical analyses of plasma in fed and fasted mice were performed. Those have demonstrated that consistent with induced by fasting lipolysis, 
a

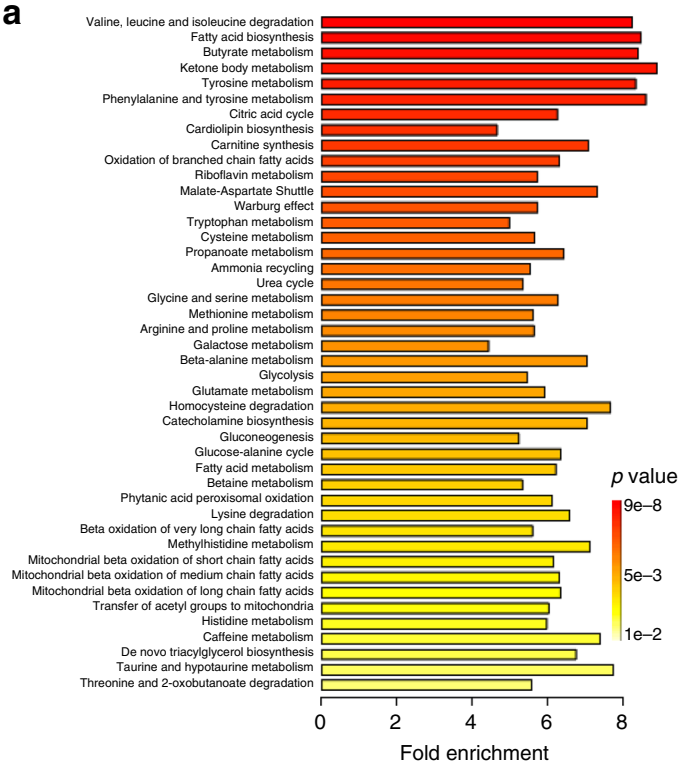

d

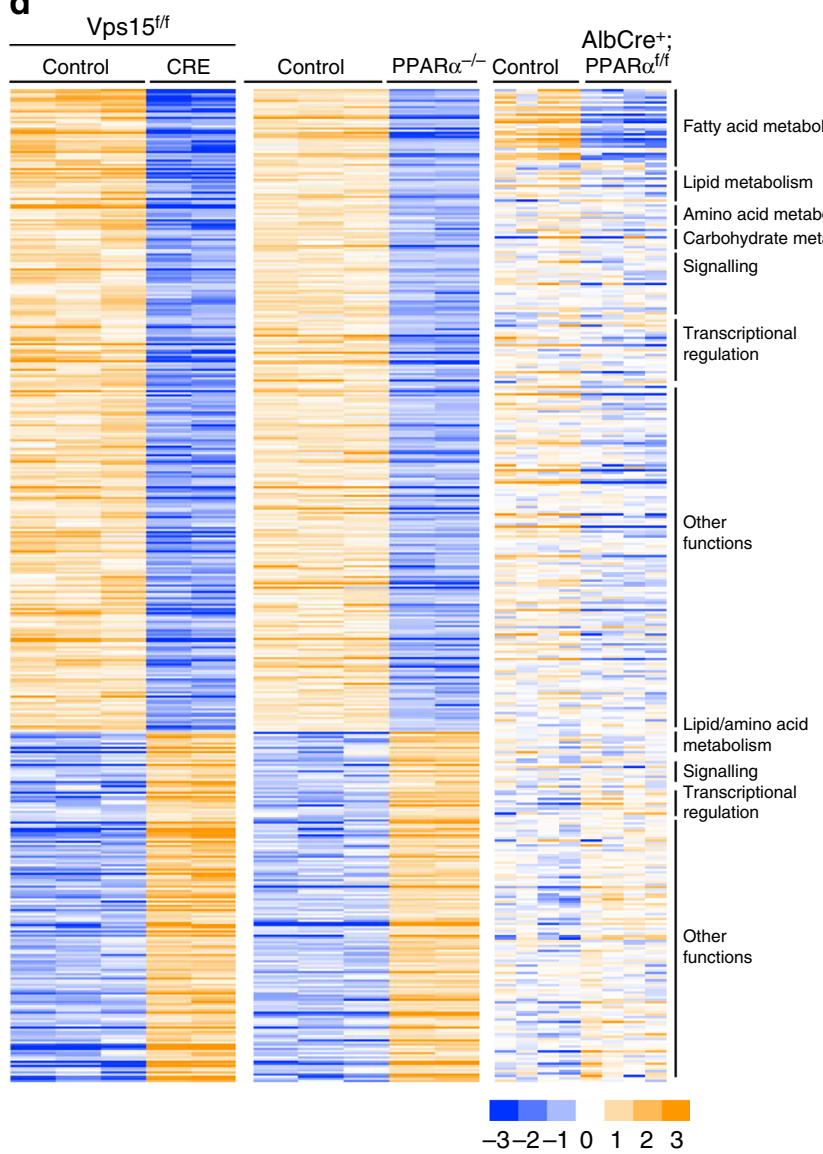

b


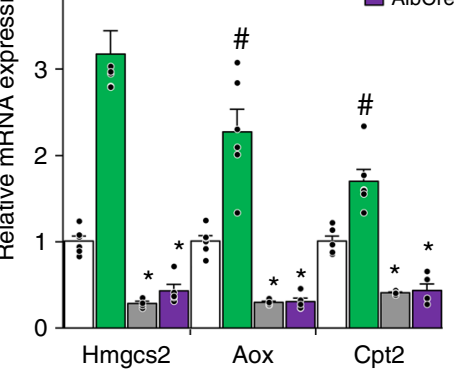

f



g

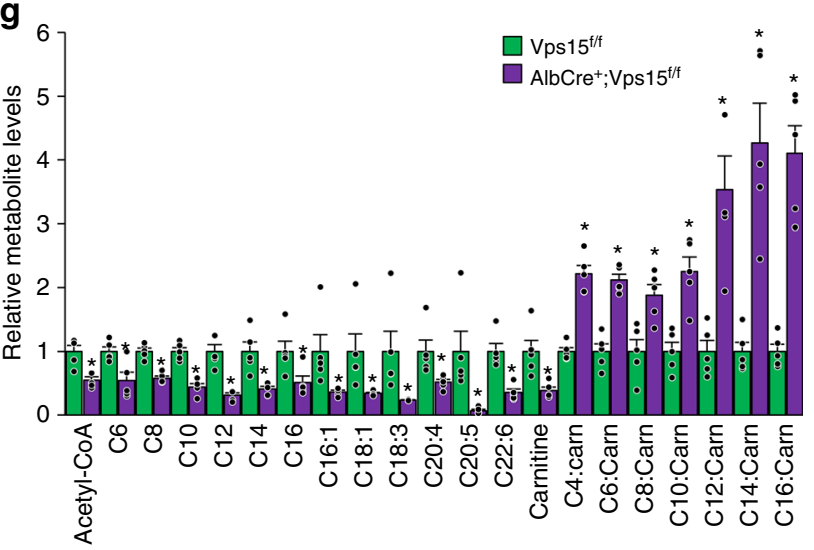

contrary, the lactate levels were decreased albeit did not reach statistical significance in the plasma of fed Vps15-LKO mice ( $P$ value of 0.059 , unpaired two-tailed Student's t-test) and fasting further lowered it in both genotypes (Supplementary Fig. 4e). Adipose tissue lipolysis is a major source of circulating fatty acids in fasting. Consistent, 24-h starvation resulted in a decrease in whole body adipose tissue content measured by dual energy X-ray absorptiometry (DEXA) scan in control and Vps15-LKO mice. In

the levels of circulating glycerol and non-esterified free fatty At the same time, consistent with effective uptake by liver, the plasmatic levels of triglycerides were decreased in fasted wild-type animals (Supplementary Fig. 4e). Notably, in Vps15-LKO mice, the levels of non-esterified free fatty acids and triglycerides were higher in fed state with fasting further increasing only the levels of non-esterified free fatty acids (Supplementary Fig. 4e). On the 
Fig. 2 Defective PPAR $\alpha$ and fatty acid oxidation in liver of Vps15 mutant. a Summary plot for metabolite set enrichment analysis (the colour of the bar indicates the significance from red to yellow). All identified metabolites are listed in Supplementary Data 3. Liver tissue of six week old $V p s 15^{f / f}(n=5)$ and AlbCre $; \operatorname{Vps} 15^{f / f}(n=4)$ mice was collected for analyses after 6-h fasting. $\mathbf{b}$ Heat map showing relative levels of the fatty acids and Acyl-carnitine derivatives measured by mass spectrometry in mouse liver collected as in a. Each column corresponds to a different $\mathrm{Vps} 15^{f / f}$ and $A l b C r e+; \mathrm{Vs} 15^{f / f}$ animal and the colour of the cell indicates the relative content of the metabolite (from blue to red). $P<0.05^{\star}$ : vs $\vee p s 15^{f / f}$. c Hydroxybutyrate levels in plasma of six week old $\mathrm{Vps} 15^{\mathrm{f} / \mathrm{f}}$ and AlbCre ${ }^{+} ; \mathrm{Vps} 15^{\mathrm{f} / \mathrm{f}}$ mice that were treated as in $\mathbf{a}$. Data are means $\pm \mathrm{SEM}\left(n=5\right.$ for $\mathrm{Vps} 15^{\mathrm{f} / \mathrm{f}}, n=4$ for $\mathrm{AlbCre}{ }^{+} ; \mathrm{Vps} 15^{\mathrm{f} / \mathrm{f}}, P<0.05^{*}: \mathrm{vs}$ $V p s 15^{f / f}$, two-tailed, unpaired Student's $t$ test). $\mathbf{d}$ Heat map representing data from a gene expression profiling experiments performed with liver samples of

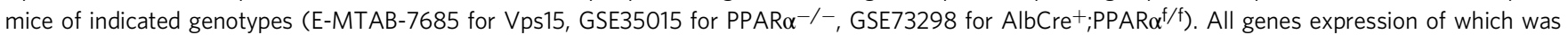
significantly modified were included in the analyses. The colour of the cell indicates the relative change of expression (from blue to yellow). The genes are grouped according to their biological function in GO annotation. The gene names corresponding to GO groups are listed in Supplementary Data 4. e Relative transcript levels of rate limiting enzymes in fatty acid transport to mitochondria, ketogenesis and FAO in the livers of 6 week old random-fed or 24-h fasted

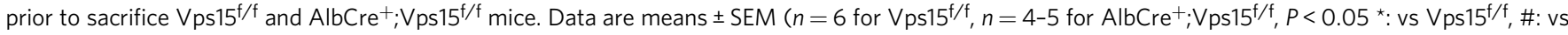
fed, two-tailed, unpaired Student's $t$ test). $\mathbf{f} I$ mmunoblot analysis of nuclear protein liver extracts of random-fed six week old $V p s 15^{f / f}$ and $A l b C r e^{+} ; V_{p s} 15^{f / f}$ using indicated antibodies. Immunoblot with LaminA/C antibody served as a loading control. Densitometric analyses of protein levels normalised to

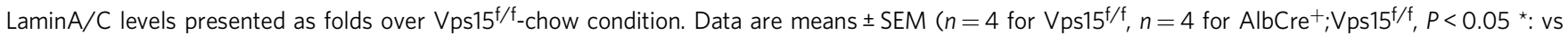
$V p s 15^{f / f}$, two-tailed, unpaired Student's $t$ test). g Relative levels of free fatty acids, carnitine and Acyl-carnitine in livers of $24-\mathrm{h}$ fasted $V p s 15^{f / f}$ and AlbCre ; $\vee p s 15^{f / f}$ mice measured by mass spectrometry. Data are means \pm SEM $\left(n=5, P<0.05^{*}\right.$ : vs $\vee p s 15^{f / f}$, two-tailed, unpaired Student's $t$ test $)$

line with important dyslipidaemia, Vps15-LKO mice showed decreased adipose tissue content already in fed state which was further reduced by fasting (Supplementary Fig. 4f). This decreased adiposity in Vps15-LKO mice was paralleled by significantly increased lean mass and no changes in body fluid levels (Supplementary Fig. $4 \mathrm{~g}$ and $4 \mathrm{~h}$ ). Moreover, despite presented with decreased adiposity, the fasting induced loss of fat was higher in Vps15-LKO as compared to wild-type mice albeit did not reach statistical significance ( $\mathrm{P}$ value of 0.056 , unpaired twotailed Student's $t$-test) (Supplementary Fig. 4i). Finally, consistent with the lower lipid levels measured in liver of Vps15-LKO mice, the mass spectrometry analyses further confirmed a decrease in free fatty acids, including unsaturated fatty acids, in livers of fasted Vps15-LKO mice (Fig. 2g). Similar to analyses in six-hour fasted mice, acyl-carnitine derivatives were accumulated in liver tissue of 24-h fasted and in fed Vps15-LKO animals (Fig. 2g and Supplementary Fig. 4j). These analyses also showed comparable levels of free carnitine in livers of fed mice, however, those were significantly decreased in livers of fasted Vps15-LKO compared to fasted wild-type mice (Fig. 2g and Supplementary Fig. 4j). Altogether, these findings suggest that hepatic expression of Vps15 is required for whole body lipid homeostasis. Furthermore, in liver, Vps15 acts upstream of PPARa and is necessary for its expression and transcriptional activity to assure efficient fatty acid degradation during fasting.

Fenofibrate treatment restores lipid catabolism in Vps15-LKO mice. The lack of endogenous PPARa ligands is a likely explanation for the dysfunction of PPARa in Vps15-LKO mice. The available selective synthetic ligands of PPAR $\alpha$, like fenofibrate, are efficient in pharmacotherapy of hyperlipidaemias by promoting fatty acid uptake, transport and oxidation ${ }^{45}$. Given that residual PPARa protein was expressed in livers of Vps15-LKO mice (Fig. 2f), we hypothesized that fenofibrate treatment could be efficient in restoring PPAR $\alpha$ transcriptional activity and correcting metabolic defects in the Vps 15 hepatic mutant. To this end, Vps15-LKO mice were treated during two weeks with fenofibrate incorporated in food. This short-term treatment normalized triglyceride levels in the plasma of Vps15-LKO mice (Supplementary Fig. 5a). Importantly, fenofibrate treated control mice induced a robust increase in transcript and protein expressions of key PPAR $\alpha$ target genes involved in fatty acid transport and catabolism (Fig. 3a, b). These include PPARa targets for peroxisomal function (Pex5 and Pex6), fatty acid transport (Fabp1 and Cpt1), ketogenesis and FAO (Hmgcs2, Lcad and
Aox). Importantly, in Vps15-LKO mice, administration of the synthetic ligand was sufficient to restore PPARa transcriptional activity, at least to levels observed in chow-fed control mice (Fig. 3a, b). Consistent with its transcriptional activation, nuclear levels of PPAR a as well as Fabp1 protein, which is required for delivery of PPAR $a$ ligands, were induced by fenofibrate treatment (Fig. 3c; Supplementary Fig. 5b). Therefore, administration of synthetic ligand restores PPAR $\alpha$ transcriptional activity in liver of Vps15-LKO mice.

Next, to address whether transcriptional activation of PPAR $\alpha$ by fenofibrate was sufficient to rescue the lipid oxidation in livers of Vps15-LKO mice, we analysed relative metabolite levels by mass spectrometry. As expected, fenofibrate administration resulted in a increase of short-chain and sharp decrease of long-chain fatty acid carnitine derivatives in the livers of control mice (Fig. 3d). Notably, this two-week fenofibrate treatment increased Acetyl-CoA and normalized levels of long-chain fatty acid carnitine derivatives in livers of Vps15-LKO mice (Fig. 3d). Thus, pharmacologic activation of PPARa by a synthetic ligand is sufficient to rescue defective lipid oxidation in livers of Vps15LKO mice.

PPARa repressors are accumulated in livers of Vps15-LKO mice. The defective PPARa transcriptional responses in livers of Vps15-LKO mice concurred with the nuclear accumulation of its bona fide repressors, Hdac3 and NCoR1 (Fig. 3c). It was also evident at total protein level in extracts of liver tissue of Vps15LKO mice and increased complex formation of PPARa with NCoR1 protein detected in liver extracts of Vps15 mutants (Supplementary Fig. $5 \mathrm{c}$ and $5 \mathrm{~d}$ ). Notably, there were no difference in NCoR1 and Hdac3 transcript levels between the control and Vps15 mutant (Supplementary Fig. 5e). Moreover, fenofibrate treatment was sufficient to reduce nuclear levels of Hdac3 and NCoR1 repressors without affecting their total protein levels in livers of Vps15-LKO mice (Fig. 3c; Supplementary Fig. 5c).

Inhibition of the class 3 PI3K by targeting Vps15 in different cell types, including hepatocytes, results in lysosomal dysfunction ${ }^{31,33}$. In line, both in vivo and ex vivo autophagic flux analyses demonstrated that the protein levels of NCoR1 and Hdac3 proteins were increased with inhibition of lysosomal activity using chloroquine (raises the lysosomal $\mathrm{pH}$ ) or leupeptin (lysosomal protease inhibitor) in livers of fasted wild-type mice unlike in Vps15 mutant (Fig. 3e; Supplementary Fig. 5f). The effective lysosomal flux under these conditions was evidenced by p62 degradation in livers of fasted mice and recovery of its levels 
a

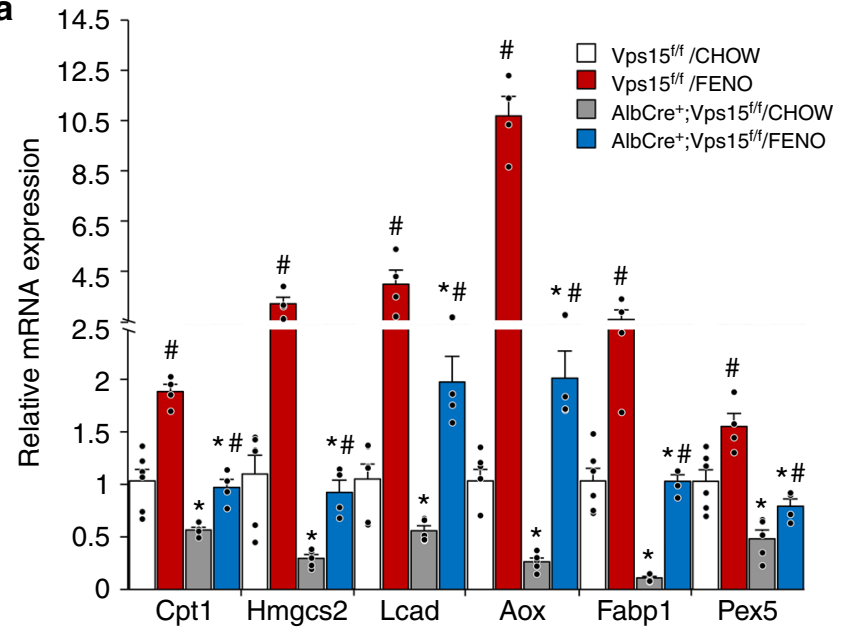

C

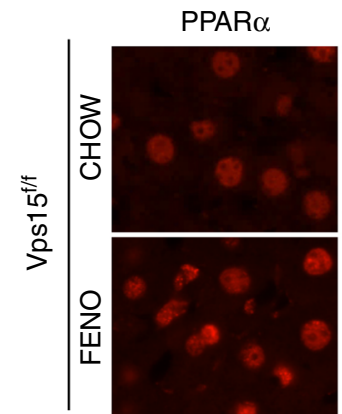

NCoR1


e

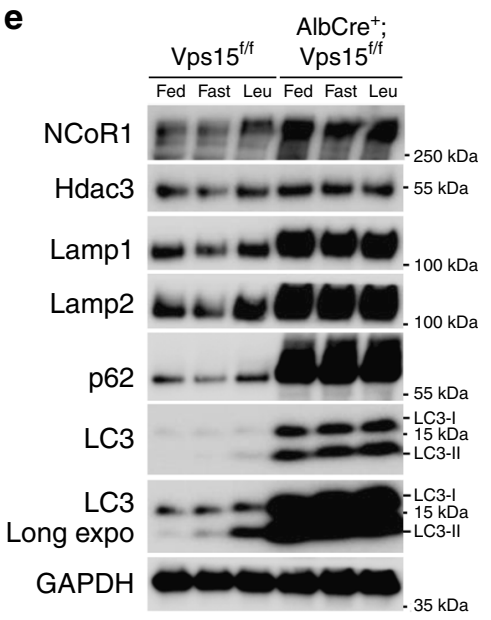

f

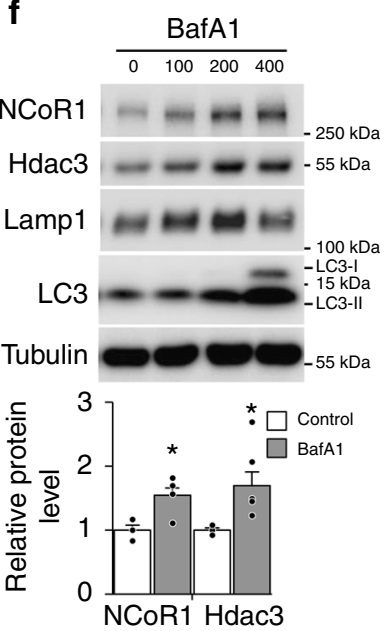

b




g



upon treatment with lysosomal inhibitors (Fig. 3e; Supplementary Fig. 5f). In addition, starvation either in vivo or ex vivo promoted lipidation of LC3 protein, signifying autophagy induction, a response that was further augmented when lysosomal activity was inhibited (Fig. 3e; Supplementary Fig. 5f). In line with blocked autophagic flux in livers of Vps15-LKO mice, LC3 and p62 proteins were highly accumulated and their levels were not further modified by those treatments (Fig. 3e; Supplementary Fig. 5f). These observations in liver tissue were further confirmed by findings in primary hepatocytes. Likewise, the inhibition of 
Fig. 3 PPAR $\alpha$ activation by fenofibrate restores lipid catabolism in Vps15-LKO mice. a Relative transcript levels of metabolic enzymes in ketogenesis, FAO, fatty acid transport and peroxisome biogenesis in livers of fed $\mathrm{Vps} 15^{\mathrm{f} / \mathrm{f}}$ and $\mathrm{AlbCre}{ }^{+} ; \mathrm{Vps} 15^{\mathrm{f} / \mathrm{f}}$ mice that were treated for two weeks with fenofibrate. Data are means $\pm \operatorname{SEM}\left(\operatorname{Vps} 15^{f / f}\left(n=6\right.\right.$ and $n=4$ for chow and FENO group), AlbCre ${ }^{+} ; \operatorname{Vps} 15^{f / f}\left(n=5\right.$ and $n=4$ for chow and FENO group), $P<0.05{ }^{\star}$ : vs $V p s 15 / f$, \#: vs chow, two-tailed, unpaired Student's $t$ test). $\mathbf{b}$ Immunoblot analysis of total protein liver extracts of mice treated as in $\mathbf{a}$ using indicated antibodies. Densitometric analyses of protein levels normalised to Pras40 levels presented as folds over $V p s 15^{f / f}$-chow condition. Data are means $\pm \mathrm{SEM}$ ( $n=4$ for $V p s 15^{f / f}$ chow and FENO group, $n=3$ for AlbCre ${ }^{+} ; V_{p s} 15^{f / f}$ chow and $n=4$ for AlbCre ${ }^{+} ; V_{p s} 15^{f / f}$ FENO group, $P<0.05^{*}$ : vs $\vee p s 15^{f / f}$, \#: vs chow, two-tailed, unpaired Student's $t$ test). c Representative images of immunofluorescent analyses showing nuclear localization of PPAR $\alpha, N C o R 1$ and Hdac3 in liver tissue of $\mathrm{Vps} 15^{\mathrm{f} / \mathrm{f}}$ and AlbCre ${ }^{+}, \mathrm{Vps} 15^{\mathrm{f} / \mathrm{f}}$ mice treated as in a. Secondary anti-mouse or anti-rabbit lgG Alexa Fluor 568 antibody were used for detection. Scale bar: $40 \mu \mathrm{m}$. d Relative levels of Acetyl-CoA and Acyl-carnitine metabolites measured by mass spectrometry in liver tissue of mice treated as in a. Data are means \pm SEM (Vps15f/f $\left(n=4\right.$ for chow and FENO group), AlbCre ${ }^{+} ; \operatorname{Vps} 15^{f / f}\left(n=3\right.$ and $n=4$ for chow and FENO group, $P<0.05{ }^{*}$ : vs $\mathrm{Vps} 15^{\mathrm{f} / \mathrm{f}}$, \#: vs chow, two-tailed, unpaired Student's $t$ test). e Immunoblot analyses in total protein liver extracts of 6-week old $\mathrm{Vps} 15^{\mathrm{f} / \mathrm{f}}$ and $\mathrm{AlbCre}^{+}$; $\mathrm{Vps} 15^{\mathrm{f} / \mathrm{f}}$ mice. Mice were either fed or fasted for $24 \mathrm{~h}$. Four hours prior the sacrifice fasted mice were injected with leupeptin (40 mg/kg) or vehicle. The total protein extracts were immunoblotted with indicated antibodies. $\mathbf{f}$ Immunoblot analysis of cytosolic protein fractions of primary hepatocytes that were grown for $72 \mathrm{~h}$ in fasting media and treated for $24 \mathrm{~h}$ before collection with increasing doses of BafA1. Densitometric analyses of protein levels normalised to Tubulin levels presented as folds over vehicle-treated cells. Data are means \pm SEM ( $n=4$ with $100 \mathrm{nM}$ Bafilomycin A1 treatment, $P<0.05$ *: vs vehicle, two-tailed, unpaired Student's $t$ test). $\mathbf{g}$ Relative transcript levels (left panel) of indicated genes in primary hepatocytes incubated in control or fasting media $(72 \mathrm{~h})$ treated with or without BafA1 for $24 \mathrm{~h}$ before collection. Data are means \pm SEM $\left(n=3, P<0.05^{*}\right.$ : vs control media, \#: vs fasting media, twotailed, unpaired Student's $t$ test). The immunoblot (right panel) served as control of autophagic activity

lysosomal activity in primary hepatocytes with Bafilomycin Al, a selective inhibitor of the vacuolar proton pump, was sufficient to induce accumulation of Hdac3 and NCoR1 proteins, mimicking the observations in livers of Vps15-LKO mice (Fig. 3f). In addition, immunofluorescent analyses in livers of fasted wild-type mice showed decreased nuclear levels of Hdac3 and NCoR1 repressors (Supplementary Fig. 5g). Those were further corroborated by immunoblot analyses in liver tissue of 24-h fasted wildtype and Vps15-LKO mice. In line with transcriptional activation of PPARa by fasting, its nuclear protein levels were upregulated in livers of wild-type mice, a response largely lacking in Vps15 mutant (Supplementary Fig. 5h). This response in PPARa protein expression was paralleled by its transcript levels, consistent with auto-control of its expression (Supplementary Fig. 5i). These changes in PPARa protein were inversely correlated with nuclear Hdac3 and NCoR1 expression (Supplementary Fig. 5h). Consistent with lysosomal dysfunction in Vps15-null liver, the accumulation of NCoR1 and Hdac3 proteins was not rescued by fasting (Supplementary Fig. 5j). The inhibitory effect of lysosomal dysfunction on PPARa transcriptional activity was further evidenced by analyses in primary hepatocytes maintained in a fasting-mimicking media with or without Bafilomycin A1. Fasting-mimicking conditions led to autophagy induction, as judged by LC3 lipidation, and resulted in a potent induction of bona fide PPARa target genes in FAO (Aox) and ketogenesis (Hmgcs2) (Fig. 3g). Importantly, these transcriptional responses were abrogated by lysosomal inhibition with Bafilomycin A1 (Fig. 3g). Altogether, these observations back the hypothesis that an excess of PPARa repressors, due to their defective lysosomal degradation, and their association with PPAR $\alpha$ contribute to PPARa inhibition in Vps15-LKO mice.

PPARa transcriptional repressors are autophagy substrates. The lysosomal degradation of PPAR $\alpha$ transcriptional repressors was further corroborated by the immunofluorescent analyses showing targeting of endogenous NCoR1 and Hdac3 to lysosomal membrane protein Lamp2-positive cellular compartment (Fig. 4a). These were further supported by findings of NCoR1 protein co-localization with the lysosomal membrane protein Lamp1-positive structures under conditions of autophagic flux (Supplementary Fig. 6a). Autophagosome membrane localized proteins of Atg8-like family, gamma-aminobutyric acid receptorassociated protein (GABARAP) and LC3, act as receptors for selective autophagic degradation of autophagy substrates. Therefore, we asked whether Hdac3 and NCoR1 proteins could bind to GABARAP and LC3. The GFP-tagged GABARAP and LC3 proteins were transiently expressed in HEK293T cells, a cell line in which endogenous $\mathrm{Hdac} 3$ and $\mathrm{NCoR} 1$ proteins are expressed and which is characterized by potent autophagic responses. The pull down of GFP-tagged LC3 and GABAPAP proteins using Trap-GFP agarose retrieved endogenous p62 protein, a known interacting partner of both proteins ${ }^{46}$ (Fig. 4b). The selectivity of the assay was revealed by exclusive interaction of ULK1 with GABARAP but not with LC3 protein ${ }^{47}$ (Fig. 4b). Notably, consistent with the requirement of the lipid kinase activity of the class $3 \mathrm{PI} 3 \mathrm{~K}$ for autophagy, the binding of ULK1 and p62 with Atg8-like proteins was decreased in cells treated with the selective class 3 PI3K inhibitor, PIK-III (Fig. 4b). Importantly, both NCoR1 and Hdac3 were found in GABARAP and LC3 precipitates with higher affinity to GABARAP protein compared to LC3 protein (Fig. 4b). Notably, the binding of NCoR1 and Hdac3 to GABARAP was decreased by pharmacological inhibition of the class 3 PI3K lipid kinase with PIK-III (Fig. 4b). Inversely, Vps15 overexpression promoted the binding of NCoR1 and Hdac3 to GABARAP (Fig. 4c). In the same conditions, Vps15 expression in HEK293T cells increased PPARa levels (Supplementary Fig. 6b). Next, we asked whether Vps15 overexpression would affect the interaction of PPAR $\alpha$ and Hdac3. The co-immunoprecipitation analyses demonstrated that binding of Hdac3 to PPARa was reduced by ectopic expression of Vps15 (Fig. 4d). These biochemical analyses were further supported by the results of a proximity ligation assay which demonstrated decreased interaction of Hdac3 and PPAR $\alpha$ in Vps15-expressing cells (Fig. 4e). In sum, these findings suggest that NCoR1 and Hdac3 co-repressors of PPARa degrade by autophagy through interaction with Atg8-like proteins in class 3 PI3K-dependent manner.

Hdac inhibition rescues PPARa responses in Vps15-LKO mice. Hdac3 is the enzymatically active component of the NCoR1containing repressor complex. To further test the contribution of Hdac3 in PPARa repression and the mitochondrial dysfunction in Vps15-depleted hepatocytes, the Hdac3 levels were downregulated by expressing selective shRNA in primary hepatocytes. Acute depletion of either Vps15 or Vp34 in primary hepatocytes resulted in inhibition of mitochondrial respiration, a defect that was rescued by the co-depletion of Hdac3 (Fig. 5a and Supplementary Fig. 7a). Similarly, the knockdown of Hdac3 in Vps15null primary hepatocytes isolated from Vps15-LKO mice restored expression of PPARa targets, Aoxl and Cpt1b (Supplementary 
a

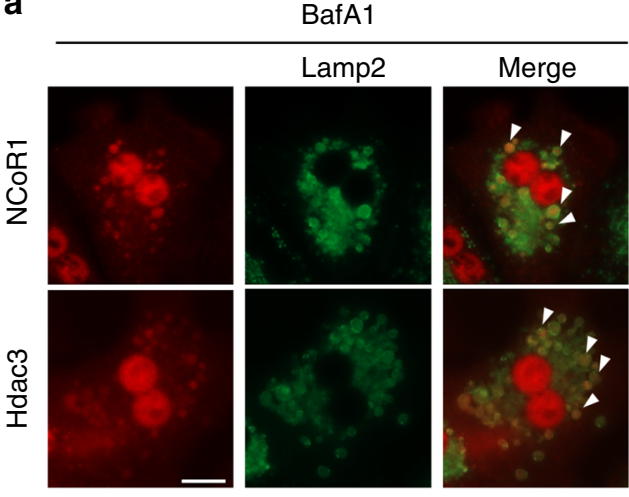

b



C



d

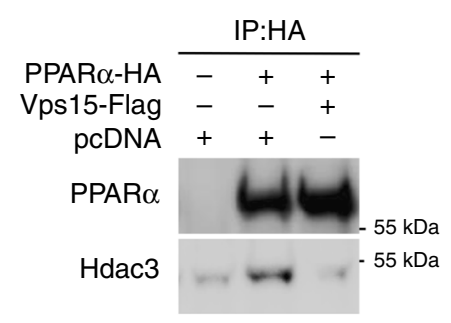

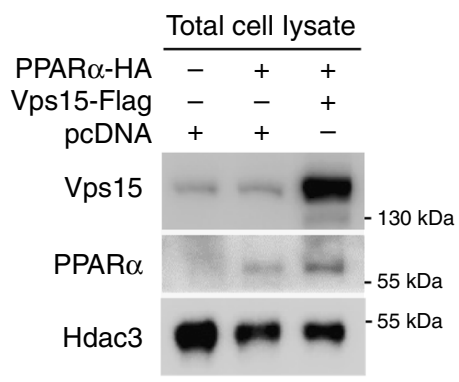

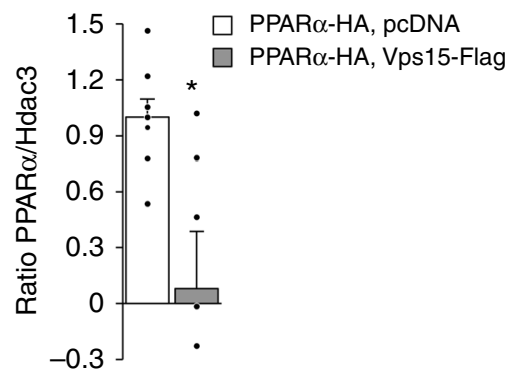

$\mathbf{e}$
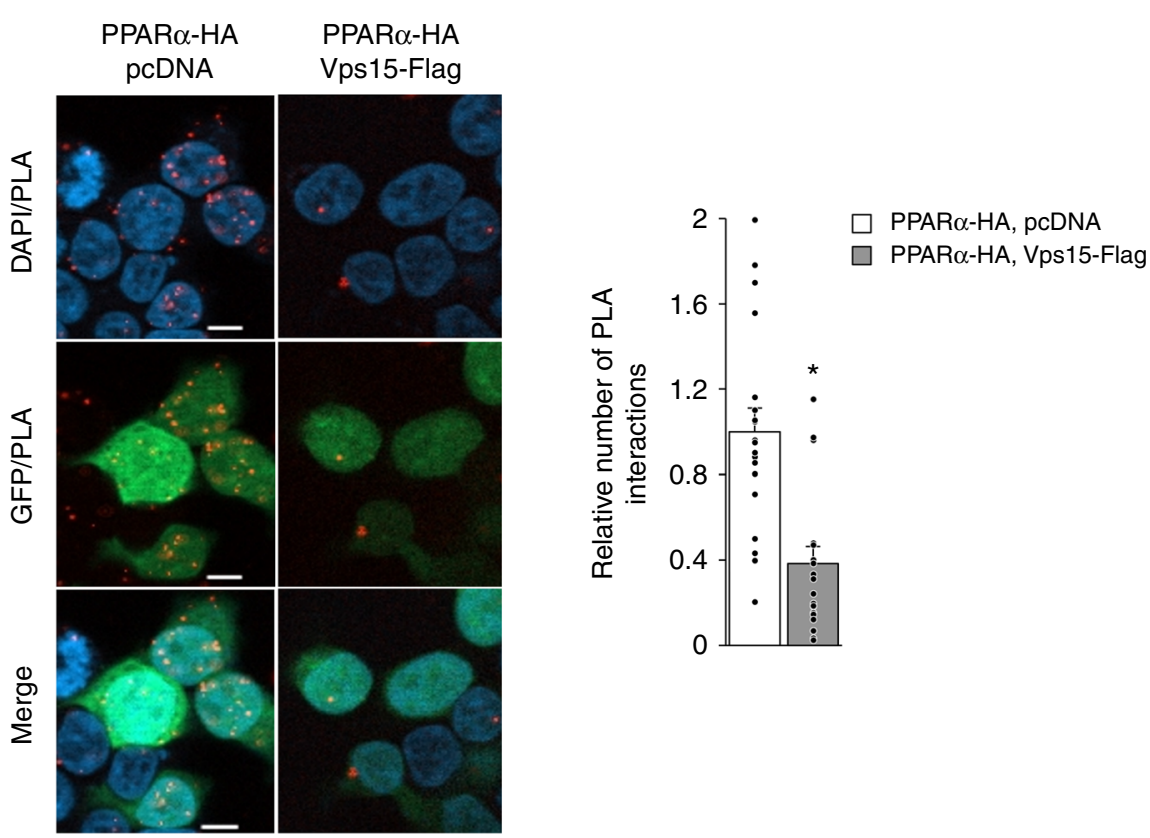

Fig. $7 \mathrm{~b}$ and $7 \mathrm{c}$ ). We extended these observations to in vivo by pharmacologically inhibiting histone deacetylases by clinically used drug valproic acid (VPA $)^{48}$. Notably, we found that in addition to Hdac3, Hdacl was also upregulated in livers of Vps15-LKO mice, further reasoning the use of the wide-range inhibitor of deacetylases, such as VPA (Supplementary Fig. 7d). The efficacy of VPA was evidenced by elevated Histone $\mathrm{H} 3$ acetylation on $\mathrm{K} 27$ in chromatin extracts of Vps15-LKO mice (Fig. 5b). Consistently, Hdac inhibition increased expression of PPARa targets in livers of the Vps15 mutant without modifying transcript or protein levels of PPARa and Hdac3 in livers of
Vps15-LKO mice (Figs. 5c, d and Supplementary Fig. 7d-7f). Importantly, VPA treatment resulted in a significant decrease of long-chain acyl-carnitine levels suggesting improved FAO in livers of Vps15-LKO mice (Fig. 5e). Altogether, these findings demonstrate that the repressive activity of Hdac3, at least partially contributes to PPARa inhibition in Vps15-LKO mice.

PPARa activation restores mitochondria mass in Vps15 mutants. Defective lipid oxidation and decreased mitochondria mass in hepatic Vps15 mutant concurred with the downregulation of the transcriptional programmes for mitochondria 
Fig. 4 Hdac3 and NCoR1 repressors degrade by autophagy. a NCoR1 and Hdac3 co-localize with Lamp2 protein in primary hepatocytes. Primary hepatocytes treated for $24 \mathrm{~h}$ with $100 \mathrm{nM}$ BafA1 were PFA fixed and co-stained with primary antibodies, secondary anti-rabbit IgG Alexa Fluor 568 and anti-rat IgG Alexa Fluor 488 antibodies were used for detection. The white arrowheads indicate double-positive cytosolic structures. Scale bar: $40 \mu$ m. $\mathbf{b}$ Immunoblot analyses of GFP-Trap agarose eluates with indicated antibodies. HEK293T cells were transfected with GFP-GABARAP, GFP-LC3 or GFP expressing plasmid constructs. Twenty-four hours post-transfection cells were treated with Vps34 inhibitor PIK-III (2.5 $\mu \mathrm{M})$ for $6 \mathrm{~h}$ before collection and proceeding with immunoprecipitation using GFP-Trap agarose. c Immunoblot analyses of GFP-Trap agarose eluates with indicated antibodies. HEK293T cells were transfected with GFP and GFP-GABARAP with or without Vps15-Flag expressing plasmid constructs. Twenty-four hours posttransfection cells were collected for immunoprecipitation using GFP-Trap agarose. d Immunoblot analyses of PPAR $\alpha$-containing complexes immunoprecipitated from HEK293T cells. HEK293T cells were transiently transfected with indicated plasmid constructs. Cells were collected $24 \mathrm{~h}$ posttransfection and PPAR $\alpha$ complexes were immunoprecipitated using anti-HA antibody. The quantification of endogenous Hdac3 protein in PPAR $\alpha$ immunoprecipitates normalized to the non-specific binding to beads is presented as fold change difference over empty vector-transfected condition. Data are means \pm SEM $\left(n=8, P<0.05^{*}\right.$ : vs empty vector-transfected cells). e Proximity ligation assay between endogenous Hdac3 and ectopic PPAR $\alpha$-HA protein in HEK293T cells co-transfected with empty vector or Vps15-Flag expressing plasmid constructs (in all conditions GFP-expressing plasmid was cotransfected). PLA-detected PPAR $\alpha /$ Hdac3 interactions (red dots) were quantified in $n=100-150$ GFP-positive cells captured on $n=19-21$ individual fields. The graph represents the number of PLA interactions as folds over empty vector-transfected condition. Data are means \pm SEM $(P<0.05 *$ : vs empty vector-transfected cells). Scale bar: $10 \mu \mathrm{m}$

biogenesis and maintenance (Fig. 1). The ultrastructural analyses demonstrated that while the number of the individual mitochondria was not changed in Vps15-null hepatocytes, the mitochondrial area was decreased in hepatocytes of Vps15-LKO mice (Fig. 6a). Notably, fenofibrate treatment was sufficient to restore mitochondrial area in Vps15-null hepatocytes (Fig. 6a). In line with the findings of fragmented mitochondria in Vps15-null hepatocytes, the immunoblot analyses in total protein extracts of liver tissue suggested activated mitochondrial fission. Specifically, the phosphorylation of small GTPase dynamin-related protein 1 (DRP1) at Ser616, a read-out of induced mitochondrial fission, was upregulated in livers of Vps15-LKO mice (Supplementary Fig. 8a). This response was induced by a 24 -h fasting in livers of wild-type mice and was constitutively upregulated in livers of Vps15-LKO mice (Supplementary Fig. 8a). It was also paralleled by stabilization and ubiquitination of Parkin E3 ubiquitin ligase that is important for selective autophagy of mitochondria (Supplementary Fig. $3 \mathrm{~d}$ and $8 \mathrm{a})^{49}$. Further immunoblot analyses have demonstrated that, consistent with induced by fasting mitochondrial fission and autophagy, Parkin and LC3-II were enriched in mitochondria fraction purified from livers of wild-type mice (Supplementary Fig. 8b). In line with mitochondrial damage, Parkin, LC3-II and poly-ubiquitinated protein levels were upregulated in purified mitochondria from livers of Vps15LKO mice and were unmodified further by fasting (Supplementary Fig. 8b). Altogether, these ultrastructural and expression analyses suggest that mitochondrial dysfunction in Vps15-LKO mice is accompanied by induced mitochondrial fission resulting in fragmented mitochondrial network. The restoration of mitochondrial mass by fenofibrate in Vps15-null hepatocytes was further corroborated by findings of increased protein expression of the mitochondrial transcription factor $(\mathrm{Tfb} 2 \mathrm{~m})$ and respiratory chain subunits (ND2, CYTB, ATP6) (Fig. 6b). These expression analyses were extended to show increased transcription of the nuclear coded mitochondria fusion protein $(M f n 2)$, mitochondria DNA replication and transcription factors (Tfam, Tfb2m, Polg), as well as their immediate downstream targets, such as respiratory chain subunits (ND1, ND2, Cytb, MTCO2, Atp6) in livers of Vps15-LKO and control mice treated by fenofibrate (Fig. 6c). Altogether, these findings suggest that PPARa, in addition to its role in transcriptional induction of FAO, might have a function in mitochondrial biogenesis and maintenance.

PGC1a rescues mitochondria activity in Vps15-null hepatocytes. The transcription factor co-activator PGC1 a plays a major role in mitochondrial biogenesis and metabolic activity by promoting the expression of nuclear encoded transcription factors and enzymes that function in mitochondria ${ }^{22}$. It also co-operates with PPARa in the activation of genes involved in $\mathrm{FAO}^{50,51}$. PGC1 $a$ expression was downregulated both at the transcript and protein level in Vps15-LKO livers (Supplementary Fig. 8c and $8 \mathrm{~d})$. In agreement with its co-activating role for PPARa, ectopic expression of $\mathrm{PGCl}$ a potently activated transcriptional activity on a conventional luciferase reporter construct that contains three tandem-repeated PPAR response elements (3xPPRE-LUC) (Fig. 6d). It also resulted in a transcriptional upregulation of a PPARa target in FAO (Cpt1) as well as mitochondria biogenesis factors Tfam and Tfb2m (Fig. 6e). Importantly, the co-expression of Vps15 with PGC1 $\alpha$ led to potentiation of these transcriptional responses (Fig. 6d, e). It was also paralleled by amplified autophagic degradation evidenced by decreased levels of bona fide autophagic substrate p62 protein and lower levels of LC3-II form as well as decreased Hdac3 levels (Supplementary Fig. 8e). This decreased Hdac3 expression was due to its lysosomal degradation as it was suppressed by inhibition of lysosomal activity with Bafilomycin A1 and Leupeptin (Supplementary Fig. 8f). Given a prominent role of PGCla in mitochondrial biogenesis, we asked whether restoration of PGC1a expression would be sufficient to rescue the mitochondrial defects in Vps15-null hepatocytes. The acute depletion of Vps15 in primary hepatocytes resulted in decreased transcript levels of key mitochondria biogenesis factors (Tfam, Tfblm and Polg) and known PPARa target Cpt1 (Fig. 6f). Importantly, adenoviral expression of PGC1a was sufficient to induce transcription of $T f a m, T f b 1 m$, Polg and Cpt1 in primary hepatocytes of wild-type mice and induced their levels in Vps15depleted hepatocytes (Fig. 6f). Furthermore, similar to the effect of Vps15 re-expression, PGC1a expression rescued the mitochondria respiration defect in Vps15-depleted hepatocytes (Fig. 6g). In sum, PGC1a is sufficient to restore mitochondrial biogenesis and activity in Vps15-null hepatocytes.

PPARa activates mitochondrial biogenesis in liver. Direct transcriptional regulation by PPAR $\alpha$ is the most likely explanation for fenofibrate's effect on mitochondrial biogenesis. In line, activation of PPARa by fenofibrate or in fasting concurred with an upregulation of mitochondrial biogenesis factors and respiratory chain subunits in the livers of control mice in PPARadependent manner (Fig. 7a and Supplementary Data 5). These observations in microarray sets were further confirmed by RTqPCR (Fig. 7b). These were in line with increased mitochondrial DNA content in livers of wild-type mice induced by fasting, a response abrogated in Vps15-LKO and PPARa-LKO mice (Fig. 7c, d). The implication of PPARa in mitochondrial biogenesis was further corroborated by findings in primary 


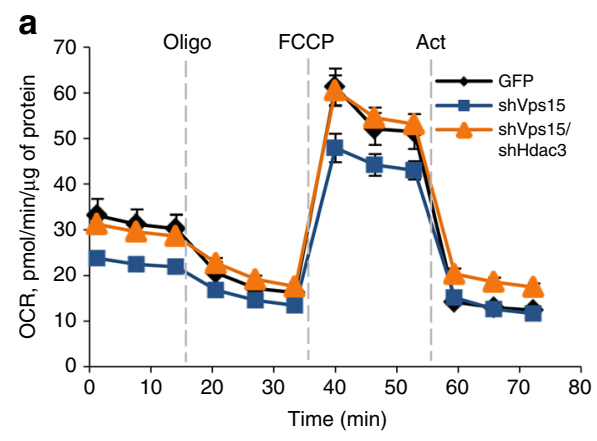

b
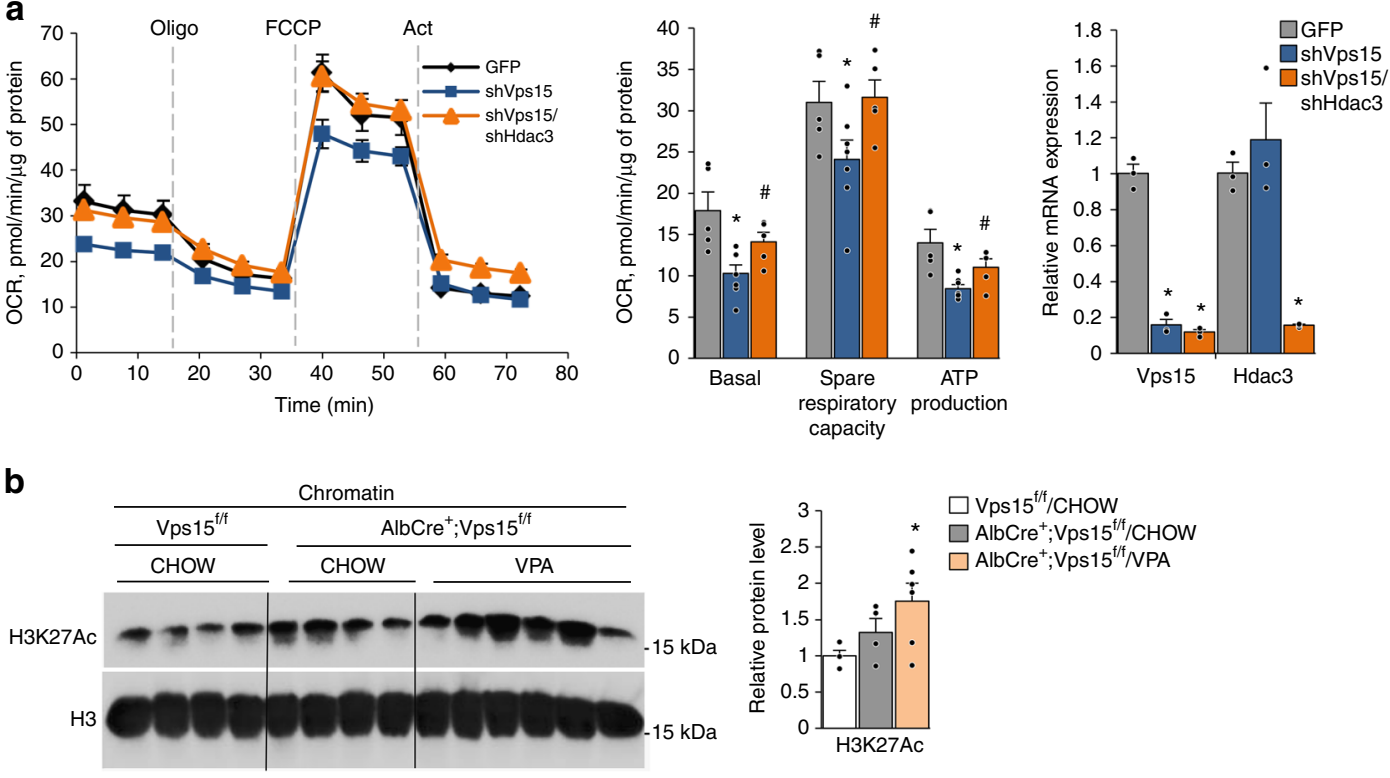

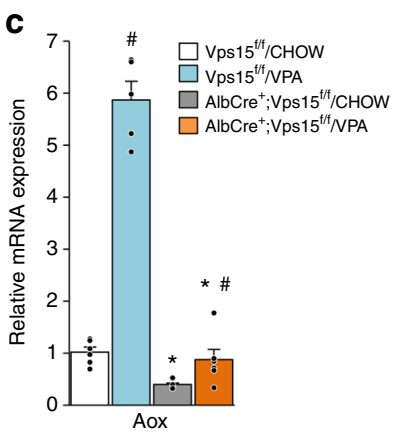

d
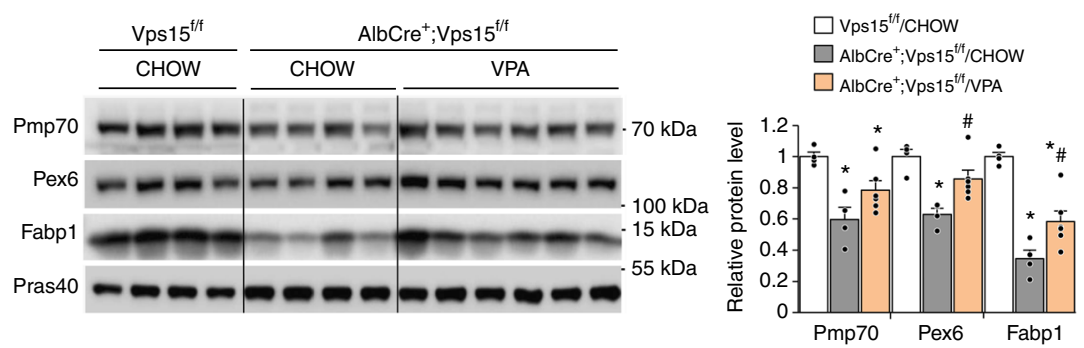

e

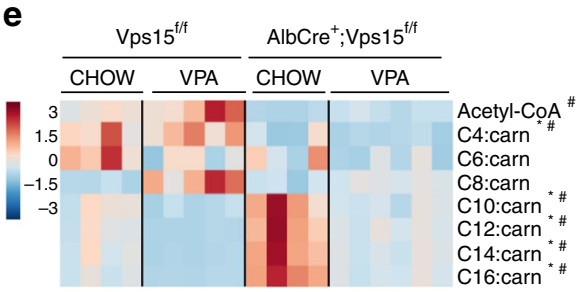

Fig. 5 Inhibition of Hdac3 improves PPAR $\alpha$ responses in livers of Vps15-LKO mice. a The OCR measured by SeaHorse Bioanalyzer in primary hepatocytes $48 \mathrm{~h}$ post-transduction with adenoviral vectors expressing GFP, shRNAVps15 or shRNAVps15 + shRNAHdac3 under basal conditions and in response to sequential treatment with indicated reagents. Dashed lines indicate the time of the addition of each reagent. Representative experiment of three independent hepatocyte cultures is presented. Quantification of basal respiration, ATP production and maximal respiratory capacity are shown on the graphs (middle panel). Relative transcript levels of Vps15 and Hdac3 is shown on right panel. Data are means \pm SEM $(n=3, P<0.05$ *: vs GFP-infected cells, \#: vs shRNAVps15-infected cells, two-tailed, unpaired Student's $t$ test). $\mathbf{b}$ Immunoblot analysis of chromatin extracts from the liver tissue of six week old random-fed $\mathrm{Vps} 15^{\mathrm{f} / \mathrm{f}}$ and $\mathrm{AlbCre}{ }^{+} ; \mathrm{Vps} 15^{\mathrm{f} / \mathrm{f}}$ mice that were treated for two weeks with VPA incorporated in food or control chow food. Densitometric analyses of H3K27Ac levels normalised to total H3 levels presented as folds over Vps15f/f-chow condition. Data are means \pm SEM $\left(n=4\right.$ for $V p s 15^{f / f}$ and AlbCre ${ }^{+} ; \mathrm{Vps} 15^{f / f}$ chow, $n=6$ AlbCre $^{+} ; \mathrm{Vps} 15^{f / f}$ VPA, $P<0.05^{*}:$ vs $\vee p s 15^{f / f}$, \#: vs chow, two-tailed, unpaired Student's $t$ test). c Relative mRNA expression levels of Aox in the livers of mice treated as in $\mathbf{b}$. Data are means \pm SEM ( $n=6$ for $V p s 15^{f / f}$ and AlbCre $;$, Vps $15^{f / f}$ chow, $n=5$ Vps15 $/ f$ VPA, $n=6$ AlbCre ${ }^{+}$; Vps15f/f VPA, $P<0.05^{*}$ : vs Vps15/f, \#: vs chow, two-tailed, unpaired Student's $t$ test). $\mathbf{d}$ Immunoblot analysis of total protein liver extracts of mice treated as in $\mathbf{b}$ using indicated antibodies. Densitometric analyses of protein levels normalised to Pras40 levels presented as folds over $V p s 15^{f / f}$-chow condition.


unpaired Student's $t$ test). e Heat map showing relative levels of Acetyl-CoA and Acyl-carnitine metabolites measured by mass spectrometry in the livers of mice treated as in $\mathbf{b}$. Each column corresponds to an individual animal and the colour of the cell indicates the relative content of the metabolite (from blue to red). Data are means $\pm \operatorname{SEM}\left(n=4\right.$ for $\mathrm{Vps} 15^{f / f}$ and AlbCre ${ }^{+} ; \mathrm{Vps} 15^{\mathrm{f} / \mathrm{f}} \mathrm{chow} n=5 \mathrm{Vps} 15^{\mathrm{f} / \mathrm{f}} \mathrm{VPA}, n=6 \mathrm{AlbCre}+\mathrm{Vps} 15^{\mathrm{f} / \mathrm{f}} \mathrm{VPA}, P<0.05^{\star}:$ AlbCre ${ }^{+}$; Vps $15^{f / f}$ vs $V p s 15^{f / f}, \#: A l b C r e^{+} ; \operatorname{Vps} 15^{f / f}$ VPA vs AlbCre ${ }^{+} ; \mathrm{Vps} 15^{f / f}$ chow, two-tailed, unpaired Student's $t$ test)

hepatocytes. As expected, incubation in fasting media stimulated expression of bona fide PPARa target genes involved in FAO (Aox), ketogenesis (Hmgcs2) and peroxisome function (Pex6) (Fig. 7e, f). Importantly, it was sufficient to promote expression of mitochondrial transcription factors and their downstream targets (Fig. 7e, f). These findings suggested that PPARa might directly activate transcription of mitochondrial biogenesis factors. To test this hypothesis, we performed a bioinformatics analysis of Tfam 

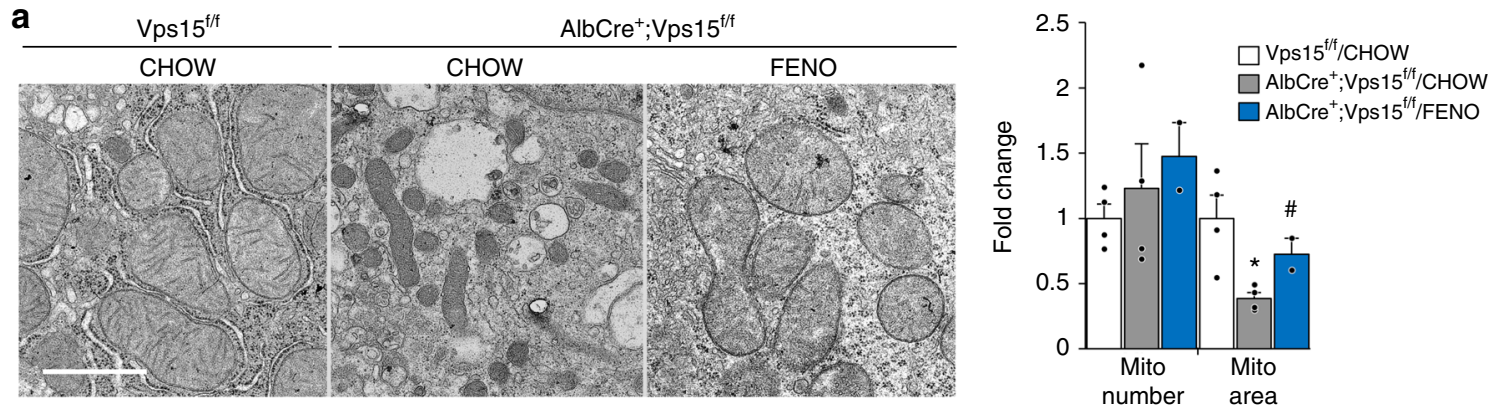

b
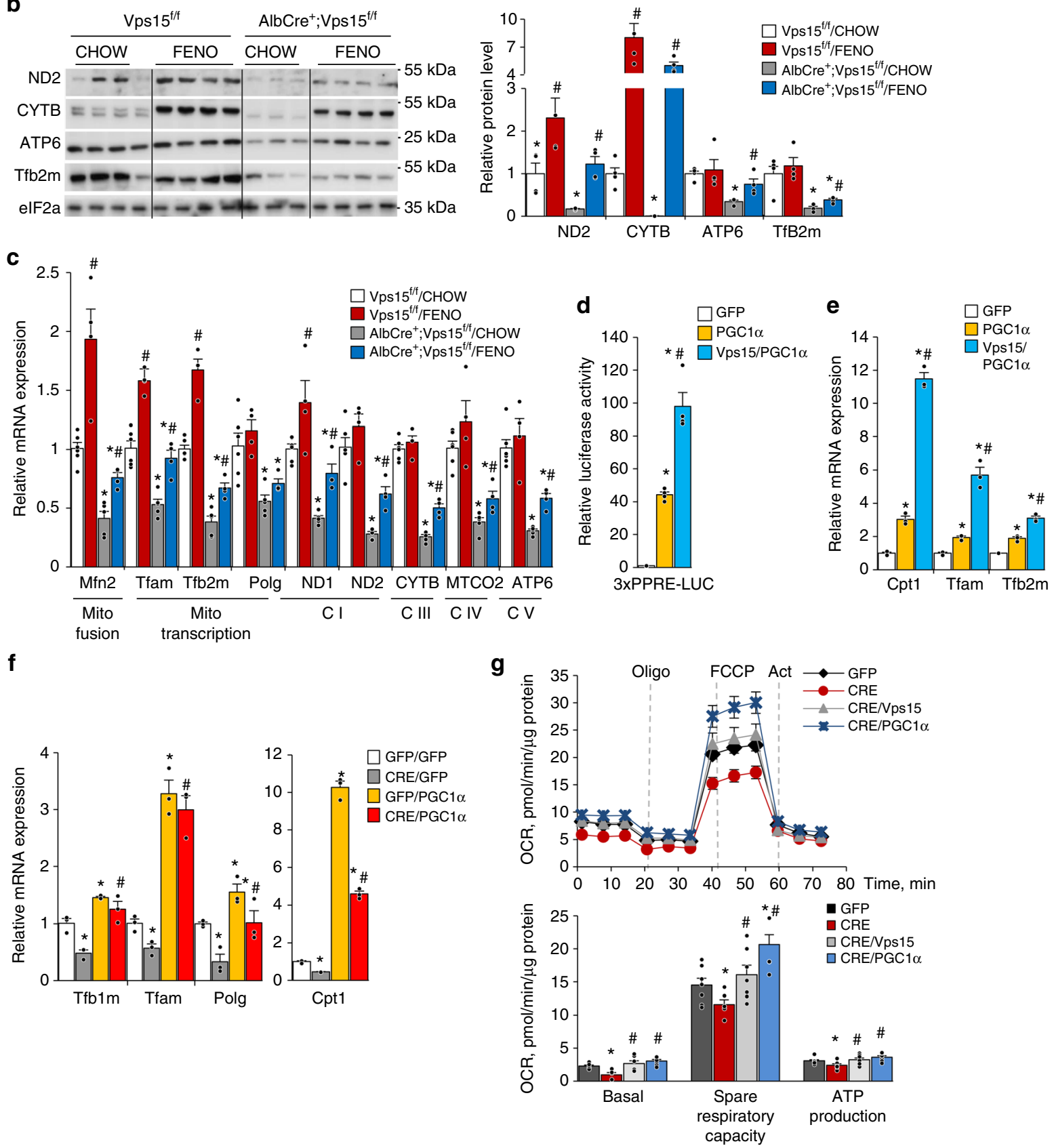

and Tfb2m gene promoters for the presence of putative PPREs. These in silico analyses identified putative PPREs that were localized in the gene of Tfam (PPRE\#1 at $-277 \mathrm{bp}$ and PPRE\#2 at $+660 \mathrm{bp}$ from the transcription start site) and in the gene of Tfb2m (PPRE\# 1 at $-361 \mathrm{bp}$, PPRE\#2 at $+181 \mathrm{bp}$ from the transcription start site) (Fig. $7 \mathrm{~g}$ ). To evaluate if PPAR $\alpha$ directly

binds to those putative PPREs, we performed chromatin immunoprecipitation (ChIP) with endogenously expressed PPARa and analyzed enrichment in PPARa binding using primers nested around the identified PPRE sequences. As shown in Fig. $7 \mathrm{~g}$, in mouse primary hepatocytes, endogenous PPAR $\alpha$ was bound to promoters of both genes and its binding was similar to a 
Fig. 6 Fenofibrate treatment rescues mitochondrial function in Vps15-LKO mice. a Representative liver transmission electron micrographs of control or fenofibrate treated $\mathrm{Vps} 15^{f / f}$ and $\mathrm{AlbCre}{ }^{+} ; \mathrm{Vps} 15^{f / f}$ mice. The fold change of relative mitochondria area and mitochondria number are presented as graph. Data are means \pm SEM of calculations of total $~ 1500$ mitochondria normalized to total area of the fields analysed $(n=15-20$ randomly taken fields, $n=2-4$ mice, $P<0.05^{*}$ : vs Vps15/f , \#: vs chow, two-tailed, unpaired Student's $t$ test). Scale bar: $1 \mu \mathrm{m}$. Protein (b) and relative transcript levels (c) of mitochondria fusion factor, mitochondrial DNA transcription factors, mitochondrial DNA polymerase and respiratory chain subunits in liver of $V p s 15^{f / f}$ and $A l b C r e+$; $\mathrm{Vps} 15^{\mathrm{f} / \mathrm{f}}$ mice treated with fenofibrate. Densitometric analyses of protein levels normalised to elF2a levels presented as folds over $V p s 15^{f / f}$-chow condition. Data are means \pm SEM ( $n=4-6$ for $V p s 15^{f / f}$ chow and FENO group, $n=4-5$ for AlbCre $;$; Vps $15^{f / f}$ chow and $n=4$ for AlbCre $;$; Vps15 ${ }^{f / f}$ FENO group, $P<$ $0.05^{*}$ : vs Vps15/f, \#: vs chow, two-tailed, unpaired Student's $t$ test). d Primary hepatocytes were transduced with adenoviral vectors expressing PGC1 $\alpha$, PGC1 $\alpha+$ Vps15 or GFP protein as a control and $24 \mathrm{~h}$ post-transduction were transfected with reporter construct. The relative luminescence presented as fold difference over empty vector transfected GFP-infected condition. Data are means \pm SEM $(n=4$ independent hepatocyte cultures, $P<0.05 *$ : vs empty vector, \#: vs PGC1 $\alpha$, two-tailed, unpaired Student's $t$ test). e Relative mRNA expression levels of indicated genes in primary hepatocytes $36 \mathrm{~h}$ posttransduction with indicated adenoviral vectors. Data are means \pm SEM (representative experiment of $n=3$ independent cultures, $P<0.05$ *: vs GFP, \#: vs PGC1 $\alpha$, 2-tailed, unpaired Student's $t$ test). f Relative transcript levels of mitochondria biogenesis factors and PPAR $\alpha$ target Cpt1 in primary Vps15f/f hepatocytes in which Vps15 was depleted by expressing Cre recombinase using adenoviral vectors. Thirty-six hours after initial infection PGC1 $\alpha$ was expressed for additional $24 \mathrm{~h}$ using adenoviral vectors. Data are means \pm SEM (representative experiment of $n=3$ independent hepatocyte cultures, $P<$ $0.05^{*}$ : vs GFP/GFP, \#: vs Cre/GFP, two-tailed, unpaired Student's $t$ test). $\mathbf{g}$ The OCR measured by SeaHorse Bioanalyzer in primary Vps $15^{f / f}$ hepatocytes in which $V p s 15$ was depleted by expressing Cre recombinase using adenoviral vectors. Thirty-six hours after initial infection, cells were transduced with adenoviral vectors to express PGC1 $\alpha$ or Vps15 for additional $24 \mathrm{~h}$. First, OCR under basal conditions and then in response to sequential treatments is shown. Representative experiment of three independent hepatocyte cultures is presented. Dashed lines indicate the time of the addition of each reagent. Quantification of basal respiration, ATP production and maximal respiratory capacity are shown on the graphs (right panel). Data are means \pm SEM $(P<$ 0.05 *: vs GFP-infected cells, \#: vs Cre-infected cells, two-tailed, unpaired Student's $t$ test)

characterized PPRE in the promoter of its own gene. In addition, chromatin immunoprecipitation analyses from liver tissue demonstrated significantly lower enrichment of transcription permissive $\mathrm{H} 3 \mathrm{~K} 27 \mathrm{Ac}$ histone mark in regions encompassing characterized PPREs in promoter of PPARA, its known target gene Fabp1 as well as PPRE in the promoter of $T f b 2 m$ gene in livers of Vps15 mutants compared to controls (Supplementary Fig. 8g). These finding were consistent with decreased transcript levels of PPARa, Fabp1 and TFb2m and accumulation of PPARa transcriptional repressors, NCoR1 and Hdac3, in livers of Vps15LKO mice. Altogether, these observations advocate that PPAR $\alpha$ in addition to its established role in fatty acid degradation directly contributes to mitochondrial biogenesis in liver (Fig. 7h).

\section{Discussion}

In growth permissive conditions with high nutrient levels, proanabolic insulin and mammalian target of rapamycin (mTOR) signalling inhibits autophagy and mitochondrial lipid catabolism $^{52}$. Mechanistically, this involves the cytosolic retention and proteasomal degradation of TFEB and PPAR $\alpha / P G C 1 \alpha$ transcription complexes as well as the control of stability, nuclear localization and recruitment to transcription factors of NCoR1 and Hdac3 repressors $38,53-55$. The positive role of TFEB and PPARa in the transcriptional control of lysosomal activity and autophagy in liver was demonstrated during physiologic fasting when nutrient-driven insulin and mTOR signalling are blunted $^{17,20}$. This synchronous activation of PPARa and PGC1a in fasting assures expression of genes involved in fatty acid transport, degradation, as well as autophagy machinery and mitochondrial biogenesis factors to match increased energy demand with the metabolic capacity of mitochondria. Notably, lysosomal pathway of autophagy is a central mechanism for the adaptation to fasting, as evidenced by perinatal lethality of autophagy deficient mice ${ }^{3,4}$. Yet, the possibility that autophagy controls at transcriptional level metabolic responses downstream of PPARa has not been addressed.

Among components of the autophagic pathway, the class 3 PI3K is ideally positioned to integrate signals from environment to adjust cellular metabolic activities depending on the energy status. Its lipid kinase activity drives autophagic flux and vesicular trafficking towards lysosomes, which are impaired in Vps15 and Vps34 tissue-specific mouse mutants ${ }^{13,31,33,56,57}$. In addition to autophagy, the class $3 \mathrm{PI} 3 \mathrm{~K}$ also assures a retrocontrol of insulin signalling by promoting lysosomal degradation of the insulin receptor in hepatocytes; and therefore, it impacts whole-body glucose homeostasis ${ }^{33}$. The findings that we present here in the context of liver tissue establish an additional feed-forward loop that couples activation of the class 3 PI3K to the transcriptional induction of autophagy, mitochondrial biogenesis and lipid catabolism downstream of PPARa. The most significant conclusions of our work are: (1) mitochondrial biogenesis and fatty acid oxidation in hepatocytes require functional class $3 \mathrm{PI} 3 \mathrm{~K}$; (2) liver-specific deletion of the regulatory subunit Vps15 phenocopies the loss of PPARa, suggesting that both genes functionally interact; (3) mechanistically, loss of the class 3 PI3K signaling in liver manifests in PPARa ligand shortage, decreased expression of its co-activator PGC1a and accumulation due to defective autophagic degradation of its transcriptional co-repressors $\mathrm{Hdac} 3$ and NCoR1. Altogether, our work demonstrates a key role of the class 3 PI3K in coordination of metabolic adaptation to fasting.

Likely, multiple mechanisms are involved in PPARa dysfunction in the class 3 PI3K mutant and future studies will establish their specific contribution. Our work suggests that, first, the defective lysosomal degradation of NCoR1 and Hdac3 repressors due to block of autophagy correlates with and probably contributes to PPARa inhibition in Vps15-LKO livers. Second, a lack of free fatty acids, which act as PPAR a ligands, likely adds to PPARa dysfunction in the Vps15 mutants. To this end, the inhibition of de novo fatty acid synthesis, a physiologically relevant source of PPARa ligands, would be consistent with the accumulation of Hdac3 and NCoR1 co-repressors which, in addition to PPARa, also inhibit pro-lipogenic liver $\mathrm{X}$ receptor (LXR) transcription factors ${ }^{58}$. Finally, the deletion of Vps15 in liver manifests in profound trafficking defects of plasma membrane receptors ${ }^{33}$. In fasting, adipose tissue derived fatty acids contribute as ligands to PPARa activation. Notably, the functional connection between mitochondria and endocytosis was suggested by a genome-wide RNAi screen of endocytosis regulators which revealed an enrichment in genes having mitochondrial-related functions ${ }^{59}$. Therefore, it is likely that in fasted Vps15-LKO mice, defective trafficking of the low-density lipoprotein receptor and lipid scavenger receptors, such as CD36, contribute to the lack of PPARa ligands delivered from periphery. 


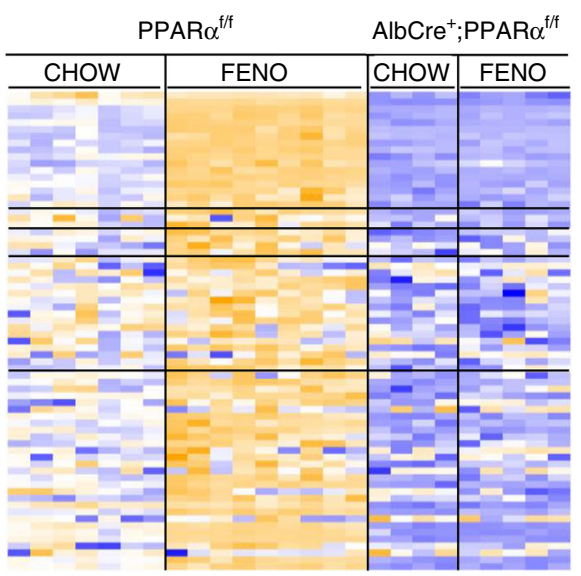

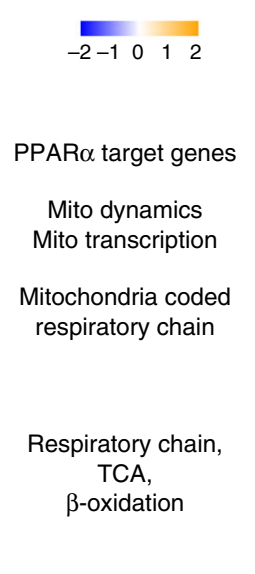

b

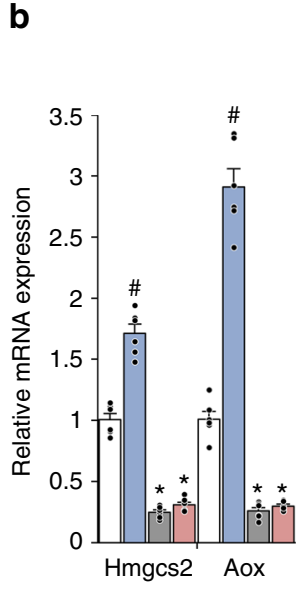

e

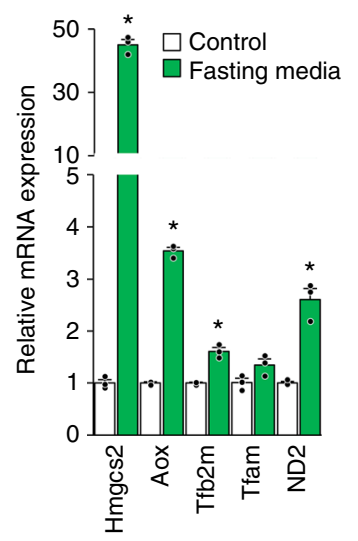



$\mathbf{f}$

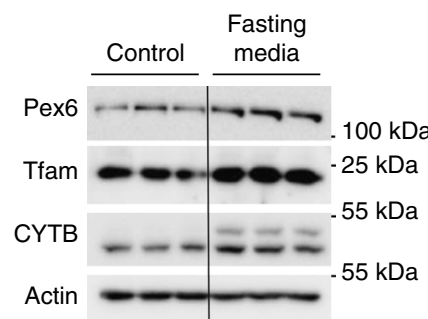

g

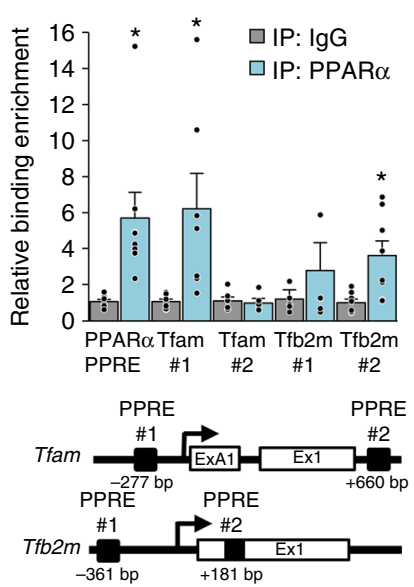

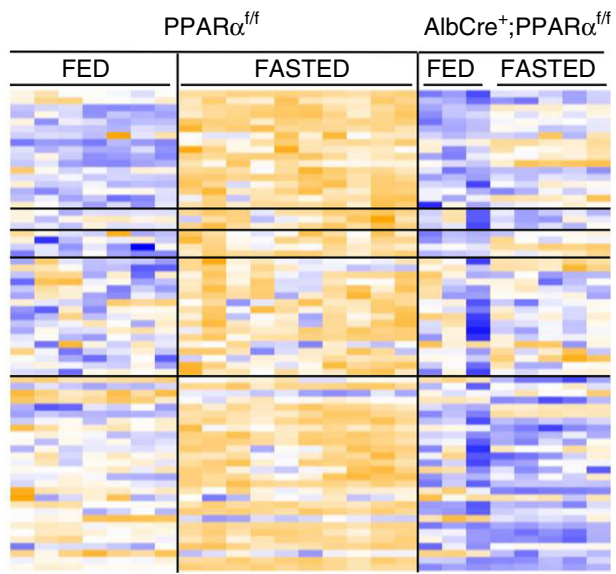

C



d

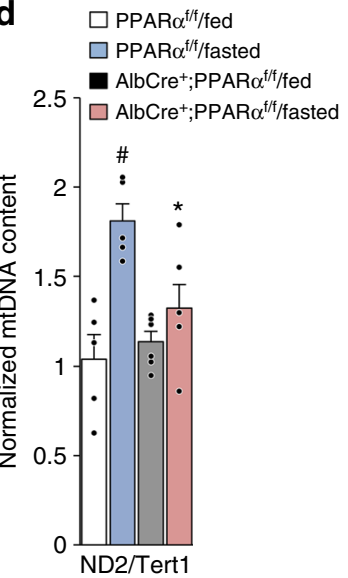

$\mathbf{h}$

h Fasting hepatocyte

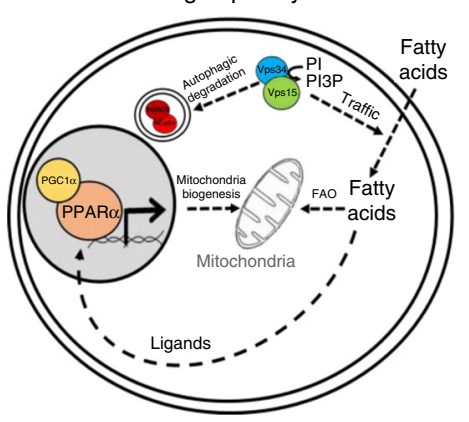

We find that hepatic PPARa, in addition to its known role in transcriptional activation of autophagy and lipid degradation, promotes mitochondrial biogenesis. This mechanism may ensure that an increased demand in the production of energy during fasting is coordinated with mitochondrial content. Notably, PGC1 a levels, similar to PPARa, are decreased in livers of Vps15LKO mice, and its expression is sufficient to restore mitochondrial dysfunction in Vps15-null hepatocytes. In addition, we cannot exclude that activated mitochondrial fission might contribute to mitochondrial dysfunction in livers of Vps15-LKO mice. Yet, likely, it is a secondary response to defective mitochondrial turnover and mitochondrial biogenesis in hepatic Vps15 mutant. The positive function of PPARa in mitochondrial biogenesis might be restricted to hepatocytes. In the heart, PPAR a activation inhibits respiratory chain gene expression, while in skeletal muscles, it promotes the metabolic transition towards glycolytic fibres which are characterised by a lower mitochondrial activity 60,61 . In skeletal muscles, deletion of PPARa reduces FAO but increases the number of oxidative fibres $^{61,62}$. Mitochondrial biogenesis in the heart and skeletal muscles is induced by a transcriptional complex of ERR and PGC1 $\alpha^{60}$. The molecular mechanisms of these tissue-specific 
Fig. 7 PPAR $\alpha$ activates mitochondrial biogenesis in liver. a Heat map representing data from a microarray experiment performed with liver samples of $\mathrm{PPAR \alpha}^{\mathrm{f} / \mathrm{f}}$ and AlbCre ${ }^{+}$PPAR ${ }^{\mathrm{f} / \mathrm{f}}$ mice treated with fenofibrate (GSE73298) or fasted for $24 \mathrm{~h}$ (GSE73299). The colour of the cell indicates the relative change of expression (from blue to yellow). The genes are grouped according to their GO annotations (biological function). The gene names corresponding to GO groups are listed in Supplementary Data 5. b Relative mRNA expression levels of mitochondrial transcription factors, respiratory chain subunits and genes of $\mathrm{FAO}$ in liver tissue of PPAR $\alpha^{f / f}$ and AlbCre ${ }^{+} ; \mathrm{PPAR}^{\mathrm{f} / \mathrm{f}}$ mice treated with fenofibrate ${ }^{37}$. Data are means \pm SEM $\left(n=6, P<0.05^{*}\right.$ : vs PPAR $\alpha^{\mathrm{f} / \mathrm{f}}$, \#: vs chow, two-tailed, unpaired Student's $t$ test). Mitochondrial versus nuclear DNA was determined by quantitative PCR analysed in total DNA purified from liver tissue of fed or 24-h fasted $\mathrm{Vps} 15^{\mathrm{f} / \mathrm{f}}$ and AlbCre ${ }^{+} ; \mathrm{Vps} 15^{\mathrm{f} / \mathrm{f}}$ mice (c) or PPAR $\alpha^{\mathrm{f} / \mathrm{f}}$ and $\mathrm{AlbCre}^{+} ; \mathrm{PPAR}^{\mathrm{f} / \mathrm{f}}$ mice $^{37}$ (d). Mitochondrial genome coded ND2 gene served as read-out of mtDNA and Tert1 as read-out of nuclear genome. Data are means \pm SEM $\left(n=4-6\right.$ for $V p s 15^{f / f}, n=4-5$ for AlbCre ${ }^{+} ; \mathrm{Vps} 15^{f / f}, n$ $=5-6$ for PPAR $\alpha^{\mathrm{f} / \mathrm{f}}$ and $\mathrm{AlbCre}{ }^{+} ; \mathrm{PPAR}^{\mathrm{f} / \mathrm{f}} ; P<0.05^{*}$ : vs wild-type mice, \#: vs fed, two-tailed, unpaired Student's $t$ test). Relative mRNA (e) and protein (f) expression levels of indicated genes in primary hepatocytes incubated for $72 \mathrm{~h}$ in control or fasting media. Data are means \pm SEM ( $n=3, P<0.05 *$ : vs control media, two-tailed, unpaired Student's $t$ test). $\mathbf{g}$ Schematic representation of putative PPRE localization in the promoter regions of mouse Tfam and Tfb2m genes (top panel). White rectangles represent exons, black rectangles-putative PPREs. Relative enrichment of endogenous PPAR $\alpha$ on putative PPREs in promoter region of Tfam and Tfb2m genes analysed by qPCR on chromatin prepared from primary hepatocytes (bottom panel). Binding of PPAR $\alpha$ to PPRE in its own promoter served as a positive control. Data are means \pm SEM, average of $n=7-8$ immunoprecipitations. $P<0.05 *$ : vs lgG, two-tailed, unpaired Student's $t$ test. $\mathbf{h}$ Proposed model depicting the class 3 PI3K which controls PPAR $\alpha$ activation for lipid degradation and mitochondrial biogenesis during fasting

roles of PPARa in mitochondrial metabolism and the involvement of the class 3 PI3K await elucidation. Finally, given their global role in control of gene expression, accumulation of NCoR1 and Hdac3 repressors in livers of Vps 15 mutants likely results in transcriptional alterations that go beyond inhibition of PPARa. Future studies will build a full molecular picture of transcriptional control downstream of class $3 \mathrm{PI} 3 \mathrm{~K}$ in maintenance of metabolic homeostasis. In conclusion, we propose that during the feedingfasting transition, the class $3 \mathrm{PI} 3 \mathrm{~K}$ orchestrates liver energy metabolism through the control of endocytosis, lysosomal pathway of autophagy and transcriptional control of mitochondrial metabolism. This makes the class $3 \mathrm{PI} 3 \mathrm{~K}$ an exquisite energy sensor that might exert transcriptional control of nuclear receptors in other organs beyond the liver. Thus, our findings could be relevant to an array of metabolic processes controlled by these ubiquitous nutrient sensors and might have a far-reaching impact in human pathophysiology.

\section{Methods}

Reagents. The following primary antibodies were used: Vps15 (1:1000, Abnova, H00030849-M03; 1:1000, Genetex, GTX108953), p62 (1:1000, Abnova, H00008878-MO1), $\beta$-actin (1:5000, Sigma, A5316), Tubulin (1:1000, Sigma, T9026), HA (1:1000, 1:200 for IF, Sigma, H9658), Pmp70 (1:500, Sigma, SAB4200181), $\beta$-catenin (1:500, BD Biosciences, 610153), Cytochrome C (BD Biosciences, 556432), Pras40 (1:1000, Cell Signaling, 2691), Ulk1(1:1000, Cell signaling, 8054S), Lamin A/C (1:1000, Cell Signaling, 2032), NCoR1 (1:1000, Cell Signaling, 5948), Hdac3 (1:1000, Cell Signaling, 85057), H3K27Ac (1:1000, Cell Signaling, 8173), H3 (1:1000, Cell Signaling, 4499), Ulk1(1:1000, Cell signaling, 8054S), Ubiquitin (1:1000, Cell Signaling, 3936), pS616 Drp1 (1:1000, Cell Signaling, 3455), Drp1(1:1000, Cell Signaling, 8570), LC3xp (1:500, Cell Signaling, 3868), LC3 (1:1000, NanoTools, 0231-100/LC3-3-5-5F10), eIF2 $\alpha$ (1:1000, Santa Cruz, sc-11386), PPARa (1:500, Santa Cruz, sc-398394, sc-9000), Tfb2m (1:500, Santa Cruz, sc-517095), GAPDH (1:1000, Santa Cruz, SC-25778), Nrf2 (1:1000, Santa Cruz, sc-13032), Parkin (1:1000, Santa Cruz, sc32282), GST (1:1000, Santa Cruz, sc-459), Pex6 (1:500, Santa Cruz, sc-271813), Fabp1 (1:500, Santa Cruz, sc50380), NCoR1 (1:500, sc-515934, Santa Cruz), Cre (1:1000, GTX127270, GeneTex), Tom40 (1:1000, ProteinTech, 18409-1-AP), ND2 (1:500, Proteintech, 19704-1-AP), Cytb (1:500, Proteintech, 55090-1-AP), ATP6 (1:500, Proteintech, 55313-1-AP), PGC1 a (1:1000, Proteintech, 66369-1-Ig), Hdac1 (1:1000, Thermo, PA1-860), Lamp1 (1:1000, Abcam, ab24170), Lamp2 (1:1000, Abcam, ab13524), GFP (1:1000, Clontech, 8362-1), Huwel (1:1000, Cell Signaling, 5695). Tube 2 agarose was from LifeSensors (UM402). GFP, GFP-Cre, Vps15 and shRNAVps15 expressing adenoviral vectors were described previously ${ }^{31}$. Adenovirus expressing shRNA Vps34 and Hdac3 were from Vector Biolabs. Adenovirus expressing PGC1 1 was kindly provided by Bertrand Blondeau. Plasmids expressing GFPLC3B and GFP-GABARAP were kindly provided by Patrice Codogno. Plasmid expressing Vps15-Flag was purchased from MRC PPU Reagents and Services. Plasmid expressing PPARa-HA was kindly provided by Urs Albrecht.

Animals. The Vsp15 conditional mutant mouse line was established at the MCI/ICS (Mouse Clinical Institute - Institute Clinique de la Souris, Illkirch, France) as described $^{31}$. Liver specific Vps15 knockout mouse line was generated as described ${ }^{33}$.
Mice were housed in specific pathogen-free conditions. Male mice (6-8 week old) were used for the experimentation. Mice were randomly allocated to experimental groups and at least three animals were used for each condition (as indicated in figure legends) to ensure statistics analyses. Animal numbers were chosen to reflect the expected magnitude of response taking into account the variability observed in previous experiments. All animal studies were performed by authorized users in compliance with ethical regulations for animal testing and research. The study was approved by the Direction Départementale des Services Vétérinaires, Préfecture de Police, Paris, France (authorization number 75-1313) and the ethical committee of Paris Descartes University (authorization number 17-052).

Treatments and metabolic studies in vivo. All animals used in the study were fed ad libitum standard chow diet (Teklad global protein diet; $20 \%$ protein, $75 \%$ carbohydrate, $5 \%$ fat $)$ and kept under $12 \mathrm{~h} / 12 \mathrm{~h}(8 \mathrm{am} / 8 \mathrm{pm})$ light on/off cycle. Animals were sacrificed between 2 and 4 pm unless indicated. For fasting experiment, the mice were food deprived for $24 \mathrm{~h}$ starting at $10 \mathrm{pm}$. For 6-h starvation for metabolomics analyses in liver tissue, the fasting was initiated at $8 \mathrm{am}$ and liver tissue collected at $2 \mathrm{pm}$. VPA $(200 \mathrm{mg} / \mathrm{kg})$ and Fenofibrate $(200 \mathrm{mg} / \mathrm{kg})$ were incorporated in chow food and mice treated during two weeks with free access to control and drug incorporated food. Body composition was assessed in all mice using DEXA scan on the minispec LF50 Mq 7.5 NMR Analyzer (Brucker) according to manufacturer's instructions. Plasmatic levels of lactate, non-esterified fatty acids, triglycerides, glycerol and hydroxybutyrate were measured enzymatically using Olympus AU 400 apparatus. TG levels in the acetone extracts of liver tissue were determined using Triglycerides FS Kit (Diasys) according to the manufacturer's instructions and as described ${ }^{63}$.

Electron microscopy. For ultrastructural analyses liver samples were prepared as described $^{64}$. Briefly, liver was perfused first with PBS and then $4 \%$ PFA before dissection. Tissue was postfixed in $2 \%$ glutaraldehyde in $0.12 \mathrm{M}$ phosphate buffer. After overnight fixation, tissues were treated with $1 \%$ osmium tetroxide in $0.12 \mathrm{M}$ phosphate buffer and embedded in epon (Fluka). Ultrathin sections $(70 \mathrm{~nm})$ were cut and stained with uranyl acetate (Plano $\mathrm{GmbH}$ ) and lead citrate (Electron Microscopy Sciences). Samples were analyzed with a transmission electron microscope (EM 902; ZEISS or CM10; Phillips) at an acceleration voltage of $80 \mathrm{kV}$ and pictures were acquired using a $2 \mathrm{~K}-\mathrm{CCD}$ (TRS Albert Tröndle) or SC200W (Orius) camera. Prior to quantification, a randomized blinded code was assigned by a separate researcher and the randomization was decoded at the time of the final data analysis.

Cell culture. Primary hepatocytes from 6 to 8 week-old mice were isolated by liver perfusion as described previously ${ }^{33}$. Hepatocytes were plated at $12 \times 10^{4} \mathrm{cells} / \mathrm{cm}^{2}$ in Williams medium (Life Technologies) supplemented with $20 \%$ FBS, $100 \mathrm{nM}$ insulin, $25 \mathrm{nM}$ dexamethasone, penicillin $(100 \mathrm{U} / \mathrm{ml})$, streptomycin $(100 \mu \mathrm{g} / \mathrm{ml})$, and amphotericin B (Fungizone) $(250 \mathrm{ng} / \mathrm{ml})$. For the infections Vps15 f/f hepatocytes $12 \mathrm{~h}$ after plating were infected with $10 \mathrm{MOI}$ of adenoviral vectors, $2 \mathrm{~h}$ after addition of viral particles the media was changed to Williams medium supplemented with a mix of antibiotics and $25 \mathrm{nM}$ dexamethasone. Cells were collected 24-48 $\mathrm{h}$ later for the analyses. For fasting-mimicking conditions, $12 \mathrm{~h}$ after plating, primary hepatocytes were washed with PBS and incubated for $72 \mathrm{~h}$ in Williams media devoid of serum and insulin, containing $50 \mu \mathrm{M}$ WY-14643, $2 \mu \mathrm{M}$ sodium octanoate, $25 \mathrm{nM}$ dexamethasone, penicillin $(100 \mathrm{U} / \mathrm{ml})$, streptomycin $(100 \mu \mathrm{g} / \mathrm{ml})$ and amphotericin B (Fungizone) $(250 \mathrm{ng} / \mathrm{ml})$. For lysosomal inhibition, primary hepatocytes were treated for $24 \mathrm{~h}$ with $100 \mathrm{nM}$ BafA1 before collection. For luciferase reporter assay, the reporter constructs were a kind gift of Dr. L. Fajas (pGL3 
empty vector and pGL3-3xPPRE). Primary hepatocytes were transduced with adenoviral vectors and $24 \mathrm{~h}$ post transduction, cells were transfected with a mix of luciferase reporters and control plasmid expressing $\beta$-Galactosidase using Lipofectamine LTX (Invitrogen). Twenty-four hours post-transfection, cells were collected for luciferase reporter activity assay as described ${ }^{65}$.

Mitochondrial activity measurements. For measurements of oxygen consumption rate by Seahorse bioanalyzer, primary hepatocytes were seeded at a density of $2 \times 10^{4}$ cells per well in a collagen coated XFe96 cell culture microplate. Twelve hours postplating cells were infected with relevant adenoviruses and mitochondrial activity was assessed at the times indicated in figure legends. Before measurement cells were balanced for $1 \mathrm{~h}$ in unbuffered XF assay media (Agilent Technologies) supplemented for OCR analysis with $2 \mathrm{mM}$ Glutamine, $10 \mathrm{mM}$ Glucose and $1 \mathrm{mM}$ Sodium Pyruvate. For OCR measurements, compounds were injected during the assay at the following final concentrations: Oligomycin (ATP synthase inhibitor to measure respiration associated with cellular ATP production, $1 \mu \mathrm{M}$ ), FCCP (uncoupling agent to measure the maximal respiration capacity; $1 \mu \mathrm{M}$ ), Rotenone and Antimycin A (ETC inhibitors to measure the non-mitochondrial respiration; $1 \mu \mathrm{M}$ ). The data were normalized to protein content measured in each well using BCA assay (Thermo Fisher Scientific) according to manufacturer's instructions. Respiratory chain enzyme activities and activity of lactate dehydrogenase were spectrophotometrically measured using a Cary 50 UV-visible spectrophotometer (Varian Inc, Les Ulis, France) as previously reported ${ }^{66}$.

Subcellular fractionation. Nuclear and cytosolic fractions were prepared using NE-PER Kit (Pierce) according to manufacturer's recommendations from $1 \times 10^{6}$ cells or $50 \mathrm{mg}$ of liver tissue. The chromatin fraction was extracted from insoluble nuclear pellet using acidic buffer as in ref. ${ }^{65}$. The crude mitochondria fraction from liver tissue was prepared as previously reported ${ }^{67}$.

Targeted metabolomics. Targeted metabolomics analyses were performed as described ${ }^{68}$. Briefly, extraction solution used was $50 \%$ methanol, $30 \% \mathrm{ACN}$, and $20 \%$ water. The volume of extraction solution added was calculated from weight of powdered tissue $(60 \mathrm{mg} / \mathrm{ml})$. After addition of extraction solution, samples were vortexed for $5 \mathrm{~min}$ at $4{ }^{\circ} \mathrm{C}$, and then centrifuged at $16,000 \times g$ for $15 \mathrm{~min}$ at $4{ }^{\circ} \mathrm{C}$ The supernatants were collected and analyzed by liquid chromatography-mass spectrometry using SeQuant ZIC-pHilic column (Merck) for the liquid chromatography separation. Mobile phase A consisted of $20 \mathrm{mM}$ ammonium carbonate plus $0.1 \%$ ammonia hydroxide in water. Mobile phase B consisted of ACN. The flow rate was kept at $100 \mathrm{ml} / \mathrm{min}$, and the gradient was $0 \mathrm{~min}, 80 \%$ of $\mathrm{B} ; 30 \mathrm{~min}$, $20 \%$ of $\mathrm{B} ; 31 \mathrm{~min}, 80 \%$ of $\mathrm{B}$; and $45 \mathrm{~min}, 80 \%$ of $\mathrm{B}$. The mass spectrometer (QExactive Orbitrap, Thermo Fisher Scientific) was operated in a polarity switching mode and metabolites were identified using TraceFinder Software (Thermo Fisher Scientific). For analyses, metabolomics data were normalized using the median normalization method. MetaboAnalyst 4.0 software was used to conduct statistical analyses and heatmaps generation, and unpaired two-sample $t$ test was chosen to perform the comparisons. The algorithm for heatmap clustering was based on the Pearson distance measure for similarity and the Ward linkage method for biotype clustering.

Microscopy. For fluorescent microscopy analyses on cells, primary hepatocytes were grown on collagen treated coverslips (Millipore). Twelve hours post-plating primary hepatocytes were fixed with 4\% PFA in PBS for $20 \mathrm{~min}$ and permeabilized with $0.1 \%$ saponin in PBS for $10 \mathrm{~min}$, followed by blocking in 3\% BSA in PBS. Slides were treated with primary antibodies overnight. Secondary antibodies used for these assays were anti-rabbit IgG Alexa Fluor 568 or anti-rat IgG Alexa Fluor 488 (Life Technologies). For fluorescent microscopy analyses of PPARa in liver tissue, liver was fixed by perfusion with $4 \% \mathrm{PFA}$ before dissection. The liver tissue samples were postfixed overnight in phosphate-buffered $10 \%$ formalin and embedded in paraffin. In total, $4-\mu \mathrm{m}$ sections were cut and processed for staining with anti-PPARa antibody. For NCoR1 and Hdac3 detection in liver, before been processed for staining, 8- $\mu \mathrm{m}$ sections of OCT preserved frozen liver tissue were fixed with $4 \%$ PFA for $15 \mathrm{~min}$. Fluorescence microscopy was performed using an inverted microscope (Zeiss Apotome 2) using 40× oil-immersion objective. The coded slides were examined in a blinded fashion. For histochemical analyses, liver tissue was fixed overnight in phosphate-buffered $10 \%$ formalin and embedded in paraffin. A total of $6-\mu \mathrm{m}$ sections were cut and processed for staining with HE.

Proximity ligation assay. Proximity ligation assays were performed on HEK293T cells (acquired from ATCC, bi-weekly tested for mycoplasma-free status) transfected either with empty vector (control cells) or plasmids expressing PPARa-HA with or without Vps15-flag. The vector expressing GFP protein was co-transfected in all conditions to quantify the interactions in transfected cells. The assays were carried out according to the manufacturer's instructions (DUO92101 Duolink $^{\circledast}$ In Situ Red Starter Kit Mouse/Rabbit, Sigma). In brief, transfected cells were fixed with $4 \%$ paraformaldehyde for $20 \mathrm{~min}$ and permeabilized with $0.1 \%$ Triton X-100 in PBS for 20 min at room temperature followed by blocking for 30 $\min$ at $37^{\circ} \mathrm{C}$. The cells were incubated with primary antibodies to Hdac3 (1:200, Cell Signalling) and to HA-tag (1:200, Sigma) for $1 \mathrm{~h}$ at $37^{\circ} \mathrm{C}$. The incubation with
PLA probe PLUS and MINUS conjugated with oligonucleotides was performed for $1 \mathrm{~h}$ at $37^{\circ} \mathrm{C}$. The terminal steps of ligation and amplification were performed at $37^{\circ}$ $\mathrm{C}$ for 30 and $90 \mathrm{~min}$, respectively. Images were acquired using an inverted microscope (Zeiss Apotome 2) using 60x oil-immersion objective.

Protein extraction, immunoblotting and immunoprecipitation. To prepare protein extract for immunoblot analysis, cells were washed twice with cold phosphate-buffered saline (PBS), scraped from the dishes in lysis buffer containing $20 \mathrm{mM}$ Tris- $\mathrm{HCl}$ (pH 8.0), 5\% glycerol, $138 \mathrm{mM} \mathrm{NaCl}, 2.7 \mathrm{mM} \mathrm{KCl}, 1 \% \mathrm{NP}-40,20$ $\mathrm{mM}$ NaF, $5 \mathrm{mM}$ EDTA, $1 \times$ protease inhibitors (Roche), $1 \times$ PhosphoStop Inhibitor (Roche). The same buffer was used to prepare protein extracts from liver tissue. Homogenates were spun at $12,000 \times g$ for $10 \mathrm{~min}$ at $4{ }^{\circ} \mathrm{C}$. For immunoprecipitation $500 \mu \mathrm{g}$ of cleared protein extract was incubated with $1 \mu \mathrm{g}$ of anti-HA (Sigma) antibody for $3 \mathrm{~h}$ at $+4{ }^{\circ} \mathrm{C}$. Then, immune complexes were pulled down using Protein G Sepharose beads (GE) during $2 \mathrm{~h}$ followed by four washes with extraction buffer. The protein complexes were eluted by boiling the beads in 1xSDS-sample buffer for $10 \mathrm{~min}$. Protein extracts or immunoprecipitate eluates were resolved by SDS-PAGE before transfer onto PVDF membrane followed by incubation with primary antibodies and HRP-linked secondary antibodies. Protein extracts were resolved by SDS-PAGE before transfer onto PVDF membrane followed by incubation with the primary antibodies and HRP-linked secondary antibodies. Immobilon Western Chemiluminescent HRP Substrate (Millipore) was used for the detection. The images were acquired on ChemiDocTM Imager (Biorad). The uncropped images of blots could be found as Supplementary Information.

GFP-trap assay. For immunoprecipitation with GFP-LC3 and GFP-GABARAP proteins, HEK293T cells were transiently transfected with plasmids expressing GFP, GFP-LC3B and GFP-GABARAP proteins using JetPei reagent (Thermo). Twenty-four hours post transfection, cells were collected and proteins extracted in lysis buffer $(10 \mathrm{mM}$ Tris, $\mathrm{pH} 7.5,150 \mathrm{mM} \mathrm{NaCl}, 0.5 \mathrm{mM}$ EDTA, $0.5 \% \mathrm{NP}-40)$ complemented with protease and phosphatase inhibitor cocktail (Pierce). Cell lysates were centrifuged at $15,000 \times g$ for $10 \mathrm{~min}$ at $+4^{\circ} \mathrm{C}$. The resulting supernatant was diluted with the dilution buffer $(10 \mathrm{mM}$ Tris, $\mathrm{pH} 7.5,150 \mathrm{mM} \mathrm{NaCl}, 0.5$ mM EDTA) to NP-40 final concentration of $0.1 \%$. The protein extracts were incubated with anti-GFP beads (GFP-Trap Chromotek) for $1 \mathrm{~h}$ at $+4^{\circ} \mathrm{C}$. Beads were collected by centrifugation and washed six times, the protein complexes were eluted by boiling the beads in $1 \times$ SDS-sample buffer for $10 \mathrm{~min}$

Real-time quantitative PCR. Total RNA was isolated from liver tissue using RNAeasy Lipid Tissue Mini Kit (Qiagen) and RNeasy Mini Kit (Qiagen) from primary hepatocytes. Single-strand complementary DNA was synthesized from 1 $\mu \mathrm{g}$ of total RNA using $125 \mathrm{ng}$ of random hexamer primers and SuperScript II (Life Technologies). RT-qPCR was performed on MX3005P instrument (Agilent) using a Brilliant III Ultra-Fast QPCR Master Mix (Agilent). The relative amounts of the mRNAs studied were determined by means of the $2^{-\Delta \Delta C T}$ method, with geometric mean of pinin, S18, cyclophilin, eIF2 $\alpha$, HUS, Ubiquitin as reference genes and control treatment or control genotype as the invariant control. The primer sequences are listed in Supplementary Table 1. For mtDNA quantification, total DNA from liver tissue was isolated using DNAeasy Blood and Tissue kit (Qiagen) according to the manufacturer's instructions.

Chromatin immunoprecipitation. Chromatin immunoprecipitation was performed with anti-PPAR $\alpha$ antibody (Santa Cruz, sc-398394) using primary hepatocytes or anti-H3K27Ac antibody (Abcam, ab4729) using liver tissue as described previously ${ }^{65}$. The relative amount of the immunoprecipitated DNA was determined by RT-qPCR using the $2^{-\Delta \Delta C T}$ method, with input DNA values for each sample as control. The primer sequences are listed in Supplementary Table 1.

Gene expression profiling and bioinformatic analyses. Total RNA was isolated from liver tissue of $\mathrm{Vps} 15^{\mathrm{f} / \mathrm{f}}$ mice transduced with adenoviral vectors expressing GFP or GFP-Cre protein using RNAeasy Lipid Tissue Mini Kit (Qiagen) according to manufacturer's protocol. Complementary RNA was synthesized, amplified and purified using the Illumina TotalPrep RNA Amplification Kit (Ambion) following the manufacturer's recommendations. Briefly, $200 \mathrm{ng}$ of RNA was reverse transcribed. After second-strand synthesis, the complementary DNA was transcribed in vitro and complementary RNA labeled with biotin-16-UTP. Labeled probe hybridization to Illumina BeadChips Mouse WG-6 v2 (Illumina) was carried out using Illumina's protocol. The Beadchips were scanned on the Illumina IScan using Illumina IScan image data acquisition software. Illumina GenomeStudio software (Illumina) was used for preliminary data analysis. The Illumina data were then normalized using the 'normalize quantiles' function in the GenomeStudio Software (Illumina). We used a threshold at 0.01 to convert 'Detection pval' into flags: flag $=$ 0 if pval $>0.01$ and flag $=1$ if $p \mathrm{val}<=0.01$. For changes at the transcriptional level, total RNAs from livers of two different conditions were compared. The group comparisons were done using Student's $t$-test on probes flagged as ' 1 ' for at least half of the samples involved in the comparison. We filtered the resulting $P$-values at $5 \%$ and fold change 1.5 . Heat map representations of $\log 2$ normalized expression data were generated using R (http://www.r-project.org/foundation/) and Treeview software (http://taxonomy.zoology.gla.ac.uk/rod/treeview.html) using the 
Spearman correlation similarity measure and average linkage algorithm. The gene list of all significantly modified genes (filtered fold change 1.5) could be found in Supplementary Data 1. GO analyses were performed using the Database for Annotation, Visualization and Integrated Discovery (DAVID) V6.8. The gene list was generated by selecting significantly modified genes in liver samples of $\mathrm{Vps} 15 \mathrm{f} / \mathrm{f}$ mice transduced with Adeno-Cre viral vectors (depleted of Vps15). For GO term analysis, the biological process, cellular component and molecular function categories were studied using the GO FAT default settings. Functional annotation clustering was performed with the default criteria at medium classification stringency.

Statistical analysis. Data are shown as means \pm SEM. The unpaired two-tailed Student's $t$-test was applied for statistical analysis. Results were considered significant in all experiments at $P<0.05$.

Reporting summary. Further information on experimental design is available in the Nature Research Reporting Summary linked to this article.

\section{Data availability}

Material and Correspondence: All data are available from the corresponding author upon reasonable request. The information and requests for resources and materials should be directed to Dr. Ganna Panasyuk (ganna.panasyuk@inserm.fr). The source data underlying all figures in the main text of the manuscript are provided as a Source Data file. The source data for microarray analyses in Vps15 depleted liver are deposited in ArrayExpress database E-MTAB-7685.

Received: 27 May 2018 Accepted: 20 March 2019

Published online: 05 April 2019

\section{References}

1. Ueno, T. \& Komatsu, M. Autophagy in the liver: functions in health and disease. Nat. Rev. Gastroenterol. Hepatol., https://doi.org/10.1038/ nrgastro.2016.185 (2017).

2. Ezaki, J. et al. Liver autophagy contributes to the maintenance of blood glucose and amino acid levels. Autophagy. 7, 727-736 (2011)

3. Komatsu, M. et al. Impairment of starvation-induced and constitutive autophagy in Atg7-deficient mice. J.Cell Biol. 169, 425-434 (2005).

4. Kuma, A. et al. The role of autophagy during the early neonatal starvation period. Nature 432, 1032-1036 (2004).

5. Efeyan, A. et al. Regulation of mTORC1 by the Rag GTPases is necessary for neonatal autophagy and survival. Nature 493, 679-683 (2013).

6. Hailey, D. W. et al. Mitochondria supply membranes for autophagosome biogenesis during starvation. Cell 141, 656-667 (2010).

7. Rockenfeller, P. et al. Phosphatidylethanolamine positively regulates autophagy and longevity. Cell Death Differ. 22, 499-508 (2015).

8. Thomas, H. E. et al. Mitochondrial complex I activity is required for maximal autophagy. Cell Rep. 24, 2404-2417 e2408 (2018).

9. Rambold, A. S., Cohen, S. \& Lippincott-Schwartz, J. Fatty acid trafficking in starved cells: regulation by lipid droplet lipolysis, autophagy, and mitochondrial fusion dynamics. Dev. Cell 32, 678-692 (2015).

10. Takagi, A. et al. Mammalian autophagy is essential for hepatic and renal ketogenesis during starvation. Sci. Rep. 6, 18944 (2016)

11. Youle, R. J. \& Narendra, D. P. Mechanisms of mitophagy. Nat. Rev. Mol. Cell Biol. 12, 9-14 (2011).

12. Masiero, E. et al. Autophagy is required to maintain muscle mass. Cell Metab. 10, 507-515 (2009)

13. Jaber, N. et al. Class III PI3K Vps34 plays an essential role in autophagy and in heart and liver function. Proc. Natl Acad. Sci. USA 109, 2003-2008 (2012).

14. Kundu, M. et al. Ulk1 plays a critical role in the autophagic clearance of mitochondria and ribosomes during reticulocyte maturation. Blood 112, 1493-1502 (2008).

15. Zhang, Y. et al. The role of autophagy in mitochondria maintenance: characterization of mitochondrial functions in autophagy-deficient $\mathrm{S}$. cerevisiae strains. Autophagy 3, 337-346 (2007).

16. Seok, S. et al. Transcriptional regulation of autophagy by an FXR-CREB axis. Nature 516, 108-111 (2014).

17. Settembre, C. et al. TFEB controls cellular lipid metabolism through a starvation-induced autoregulatory loop. Nat. Cell Biol. 15, 647-658 (2013).

18. Sardiello, M. et al. A gene network regulating lysosomal biogenesis and function. Science 325, 473-477 (2009).

19. Settembre, C. et al. TFEB links autophagy to lysosomal biogenesis. Science 332, 1429-1433 (2011).

20. Lee, J. M. et al. Nutrient-sensing nuclear receptors coordinate autophagy. Nature 516, 112-115 (2014).
21. Palmieri, M. et al. Characterization of the CLEAR network reveals an integrated control of cellular clearance pathways. Hum. Mol. Genet. 20, 3852-3866 (2011)

22. Scarpulla, R. C., Vega, R. B. \& Kelly, D. P. Transcriptional integration of mitochondrial biogenesis. Trends. Endocrinol. Metab. 23, 459-466 (2012).

23. Kim, K. H. et al. Autophagy deficiency leads to protection from obesity and insulin resistance by inducing Fgf21 as a mitokine. Nat. Med 19, 83-92 (2013).

24. Ma, D. et al. Autophagy deficiency by hepatic FIP200 deletion uncouples steatosis from liver injury in NAFLD. Mol. Endocrinol. 27, 1643-1654 (2013).

25. Backer, J. M. The intricate regulation and complex functions of the class III phosphoinositide 3-kinase Vps34. Biochem. J. 473, 2251-2271 (2016).

26. Panaretou, C., Domin, J., Cockcroft, S. \& Waterfield, M. D. Characterization of p150, an adaptor protein for the human phosphatidylinositol (PtdIns) 3kinase. Substrate presentation by phosphatidylinositol transfer protein to the p150.Ptdins 3-kinase complex. J. Biol. Chem 272, 2477-2485 (1997).

27. Devereaux, K. et al. Regulation of mammalian autophagy by class II and III PI 3-kinases through PI3P synthesis. PLoS ONE 8, e76405 (2013).

28. Kutateladze, T. G. Translation of the phosphoinositide code by PI effectors. Nat. Chem. Biol. 6, 507-513 (2010).

29. Matsunaga, K. et al. Two Beclin 1-binding proteins, Atg14L and Rubicon, reciprocally regulate autophagy at different stages. Nat. Cell Biol. 11, 385-396 (2009).

30. Zhong, Y. et al. Distinct regulation of autophagic activity by Atg14L and Rubicon associated with Beclin 1-phosphatidylinositol-3-kinase complex. Nat. Cell Biol. 11, 468-476 (2009).

31. Nemazanyy, I. et al. Defects of Vps15 in skeletal muscles lead to autophagic vacuolar myopathy and lysosomal disease. EMBO Mol. Med. 5, 870-890 (2013).

32. Zhou, X., Takatoh, J. \& Wang, F. The mammalian class 3 PI3K (PIK3C3) is required for early embryogenesis and cell proliferation. PLoS ONE 6, e16358 (2011).

33. Nemazanyy, I. et al. Class III PI3K regulates organismal glucose homeostasis by providing negative feedback on hepatic insulin signalling. Nat. Commun. 6 , 8283 (2015).

34. Bilanges, B. et al. Vps34 PI 3-kinase inactivation enhances insulin sensitivity through reprogramming of mitochondrial metabolism. Nat. Commun. 8, 1804 (2017).

35. Kersten, S. et al. Peroxisome proliferator-activated receptor alpha mediates the adaptive response to fasting. J. Clin. Invest 103, 1489-1498 (1999).

36. Leone, T. C., Weinheimer, C. J. \& Kelly, D. P. A critical role for the peroxisome proliferator-activated receptor alpha (PPARalpha) in the cellular fasting response: the PPARalpha-null mouse as a model of fatty acid oxidation disorders. Proc. Natl Acad. Sci. USA 96, 7473-7478 (1999).

37. Montagner, A. et al. Liver PPARalpha is crucial for whole-body fatty acid homeostasis and is protective against NAFLD. Gut 65, 1202-1214 (2016).

38. Blanquart, C., Barbier, O., Fruchart, J. C., Staels, B. \& Glineur, C. Peroxisome proliferator-activated receptor alpha (PPARalpha) turnover by the ubiquitinproteasome system controls the ligand-induced expression level of its target genes. J. Biol. Chem. 277, 37254-37259 (2002).

39. Zhao, Z. et al. Hepatic PPARalpha function is controlled by polyubiquitination and proteasome-mediated degradation through the coordinated actions of PAQR3 and HUWE1. Hepatology 68, 289-303 (2018).

40. Hirotani, M., Tsukamoto, T., Bourdeaux, J., Sadano, H. \& Osumi, T. Stabilization of peroxisome proliferator-activated receptor alpha by the ligand. Biochem. Biophys. Res. Commun. 288, 106-110 (2001).

41. Schupp, M. \& Lazar, M. A. Endogenous ligands for nuclear receptors: digging deeper. J. Biol. Chem. 285, 40409-40415 (2010).

42. Kliewer, S. A. et al. Fatty acids and eicosanoids regulate gene expression through direct interactions with peroxisome proliferator-activated receptors alpha and gamma. Proc. Natl Acad. Sci. USA 94, 4318-4323 (1997).

43. Chakravarthy, M. V. et al. "New" hepatic fat activates PPARalpha to maintain glucose, lipid, and cholesterol homeostasis. Cell Metab. 1, 309-322 (2005).

44. Forman, B. M., Chen, J. \& Evans, R. M. Hypolipidemic drugs, polyunsaturated fatty acids, and eicosanoids are ligands for peroxisome proliferator-activated receptors alpha and delta. Proc. Natl Acad. Sci. USA 94, $4312-4317$ (1997).

45. Staels, B. \& Fruchart, J. C. Therapeutic roles of peroxisome proliferatoractivated receptor agonists. Diabetes 54, 2460-2470 (2005).

46. Pankiv, S. et al. p62/SQSTM1 binds directly to Atg8/LC3 to facilitate degradation of ubiquitinated protein aggregates by autophagy. J. Biol. Chem. 282, 24131-24145 (2007).

47. Alemu, E. A. et al. ATG8 family proteins act as scaffolds for assembly of the ULK complex: sequence requirements for LC3-interacting region (LIR) motifs. J. Biol. Chem. 287, 39275-39290 (2012).

48. Gottlicher, M. et al. Valproic acid defines a novel class of HDAC inhibitors inducing differentiation of transformed cells. EMBO J. 20, 6969-6978 (2001)

49. Narendra, D., Tanaka, A., Suen, D. F. \& Youle, R. J. Parkin is recruited selectively to impaired mitochondria and promotes their autophagy. J. Cell Biol. 183, 795-803 (2008). 
50. Vega, R. B., Huss, J. M. \& Kelly, D. P. The coactivator PGC-1 cooperates with peroxisome proliferator-activated receptor alpha in transcriptional control of nuclear genes encoding mitochondrial fatty acid oxidation enzymes. Mol.Cell Biol. 20, 1868-1876 (2000).

51. Koo, S. H. et al. PGC-1 promotes insulin resistance in liver through PPARalpha-dependent induction of TRB-3. Nat. Med. 10, 530-534 (2004).

52. Saltiel, A. R. \& Kahn, C. R. Insulin signalling and the regulation of glucose and lipid metabolism. Nature 414, 799-806 (2001).

53. Sengupta, S., Peterson, T. R., Laplante, M., Oh, S. \& Sabatini, D. M. mTORC1 controls fasting-induced ketogenesis and its modulation by ageing. Nature 468, 1100-1104 (2010).

54. Settembre, C. et al. A lysosome-to-nucleus signalling mechanism senses and regulates the lysosome via mTOR and TFEB. EMBO J. 31, 1095-1108 (2012).

55. Jo, Y. S. et al. Phosphorylation of the nuclear receptor corepressor 1 by protein kinase B switches its corepressor targets in the liver in mice. Hepatology 62, 1606-1618 (2015).

56. Zhou, X. et al. Deletion of PIK3C3/Vps34 in sensory neurons causes rapid neurodegeneration by disrupting the endosomal but not the autophagic pathway. Proc. Natl. Acad. Sci. USA 107, 9424-9429 (2010).

57. Bechtel, W. et al. The class III phosphatidylinositol 3-kinase PIK3C3/VPS34 regulates endocytosis and autophagosome-autolysosome formation in podocytes. Autophagy 9, 1097-1099 (2013).

58. Mottis, A., Mouchiroud, L. \& Auwerx, J. Emerging roles of the corepressors NCoR1 and SMRT in homeostasis. Genes Dev. 27, 819-835 (2013).

59. Collinet, C. et al. Systems survey of endocytosis by multiparametric image analysis. Nature 464, 243-249 (2010)

60. Oka, S. et al. PPARalpha-Sirt1 complex mediates cardiac hypertrophy and failure through suppression of the ERR transcriptional pathway. Cell Metab. 14, 598-611 (2011).

61. Gan, Z. et al. Nuclear receptor/microRNA circuitry links muscle fiber type to energy metabolism. J. Clin. Invest. 123, 2564-2575 (2013).

62. Finck, B. N. et al. A potential link between muscle peroxisome proliferatoractivated receptor-alpha signaling and obesity-related diabetes. Cell Metab. 1 133-144 (2005).

63. Panasyuk, G. et al. PPARgamma contributes to PKM2 and HK2 expression in fatty liver. Nat. Commun. 3, 672 (2012).

64. Schatton, D. et al. CLUH regulates mitochondrial metabolism by controlling translation and decay of target mRNAs. J. Cell Biol. 216, 675-693 (2017).

65. Patitucci, C. et al. Hepatocyte nuclear factor 1alpha suppresses steatosisassociated liver cancer by inhibiting PPARgamma transcription. J. Clin. Invest. 127, 1873-1888 (2017).

66. Benit, P. et al. Three spectrophotometric assays for the measurement of the five respiratory chain complexes in minuscule biological samples. Clin. Chim. Acta 374, 81-86 (2006).

67. Frezza, C., Cipolat, S. \& Scorrano, L. Organelle isolation: functional mitochondria from mouse liver, muscle and cultured fibroblasts. Nat. Protoc. 2, 287-295 (2007).

68. Mackay, G. M., Zheng, L., van den Broek, N. J. \& Gottlieb, E. Analysis of Cell Metabolism Using LC-MS and Isotope Tracers. Methods Enzymol. 561, 171-196 (2015).

\section{Acknowledgements}

We are grateful to all lab members and the members of INSERM-U1151 for support, to Patrice Codogno for helpful discussions and Nathaniel Henneman and Nicolas Kuperwasser for manuscript editing; Bertrand Blondeau for Ad-PGC1 $\alpha$; Maurizio Crestani for sharing Ad-shRNA Hdac3; Philippe Noirez and Marion Falabregue for help with DEXA scan measurements; Sophie Berissi (SFR Small animal histology and morphology platform) and Sylvie Fabrega (SFR Viral vector and gene transfer platform) for technical support. This work was supported by grant from Agence National de la Recherche (ANR) to G.P. (ANR-JCJC-NUTRISENSPIK-16-CE14-0029) and European Research Council (ERC) to M.P. The research leading to these results has received funding from People Programme (Marie Curie Actions) of the European Union's Seventh Framework Programme (FP7/2007-2013) under REA grant agreement no. PCOFUND-GA-2013-609102, through the PRESTIGE programme coordinated by Campus France. A.I. was supported by ANR-JCJC-NUTRISENSPIK-16CE14-0029 and PRESTIGE programme. C.A. was supported by Boulos Foundation. H.G. was supported by Hepatokind grant from ANR. E.I.R was supported by Deutsche Forschungsgemeinschaft (SFB1218/A05)

\section{Author contributions}

A.I. and I.N. conducted most of the experiments, analysed the data, prepared the figures and contributed to the manuscript writing. C.A. performed animal experiments and interaction studies in cells and participated in data analyses. M.G. performed histological examination of liver tissue samples. N.C. performed the bioinformatic analyses of microarrays. E.R. and E.B. performed electron microscopy analyses. D.C. measured activity of respiratory chain complexes. H.G. and A.M. provided the PPARa mutants, shared reagents and expertise. G.P. and M.P. conceived the study and obtained funding. G.P. directed the work, designed the experiments, conducted experiments, analysed the data and wrote the manuscript. All authors discussed the results and commented on the manuscript.

\section{Additional information}

Supplementary Information accompanies this paper at https://doi.org/10.1038/s41467 019-09598-9.

Competing interests: The authors declare no competing interests.

Reprints and permission information is available online at http://npg.nature.com/ reprintsandpermissions/

Journal peer review information: Nature Communications thanks the anonymous reviewer(s) for their contribution to the peer review of this work. Peer reviewer reports are available.

Publisher's note: Springer Nature remains neutral with regard to jurisdictional claims in published maps and institutional affiliations.

Open Access This article is licensed under a Creative Commons Attribution 4.0 International License, which permits use, sharing, adaptation, distribution and reproduction in any medium or format, as long as you give appropriate credit to the original author(s) and the source, provide a link to the Creative Commons license, and indicate if changes were made. The images or other third party material in this article are included in the article's Creative Commons license, unless indicated otherwise in a credit line to the material. If material is not included in the article's Creative Commons license and your intended use is not permitted by statutory regulation or exceeds the permitted use, you will need to obtain permission directly from the copyright holder. To view a copy of this license, visit http://creativecommons.org/ licenses/by/4.0/.

(C) The Author(s) 2019 